

\title{
DISCLAMMIR
}

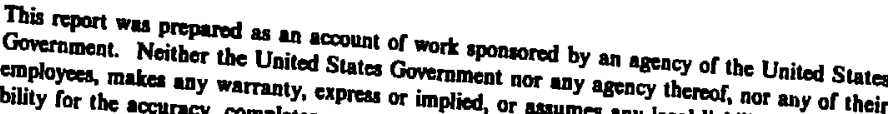
process disclosed, or reprempleteness, or usofulneas of any infor any legal liability or reaponsience herein to any specific comments its use would not infringe privatoly opparatus product, or manufacturer, or otherwise domercial product, process, or service by trade ned rights. Refermendation, or favoring by the not necessarily conatitute or imply trade name, trademark, and opinions of autho by the United States Government or imply its ondorwement, recomUnited States Govemment expresed herein do not necesenrily any agency thereof, The views

Electronics Engineering Department

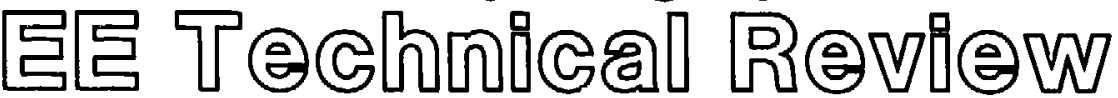

\author{
Dedicated Issue
}

\section{Distributed Computer Control System in the Nova Laser Fusion Test Facility}

\author{
Manuscript Date: September 1985
}

\section{LAWRENCE LIVERMORE NATIONAL LABORATORY University of California - Livermore, California • 94550}




\section{Preface}

The EE Technical Review has two purposes-to inform readers of various activities within the Electronics Engineering Department and to promote the exchange of ideas.

The articles, by design, are brief summaries of EE work. For further details on a subject covered, please contact cue of the authors. The authors are primarily responsible for the rontent of their articles. Inasmuch as most projects are the result of the cooperative efforts ct many individuals, the person you contact will either provide the requested information directly or refer you to the appropriate person to answer your question.

EE Department personnel are encouraged to submit articles for consideration to the Editorial Board. Board members include:

H. S. Ames-Nuclear Energy Systems Division

H. D. Francis-Field Test Systems Division

M. S. Genin-Technical Editor

K. S. Kunz-Engineering Research Division

F. D. Lee-Fusion Energy Systems Division

J. W. Spencer-Engineering Services Division

W. F. Thompson-EE Department Staff

P. J. Van Arsdall-Laser Engineering Division

E. A. Lafranchi

Electronics Engineering

Department Head 


\section{Contents}

Overview-Nova Control System

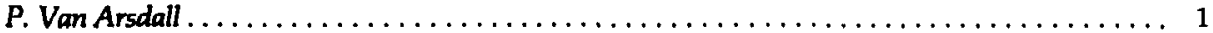

Centralized Computer-Based Controls for the Nova Laser Facility

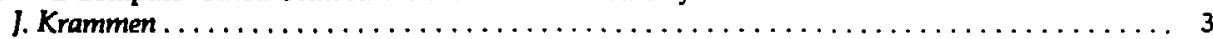

Nova Pulse-Power Control System

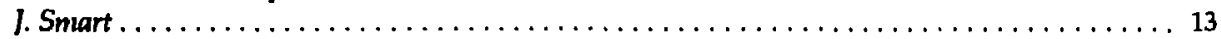

Nova Laser Alignment Control System

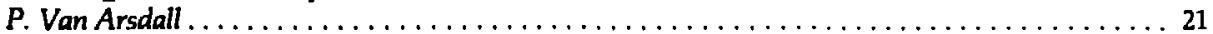

Nova Beam Diagnostic System

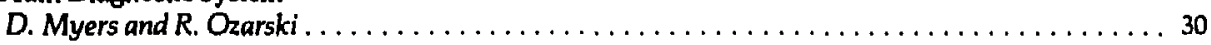

Nova Target-Diagnostics Control System

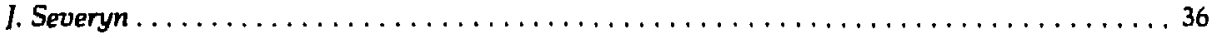

Nova Shot Scheduler

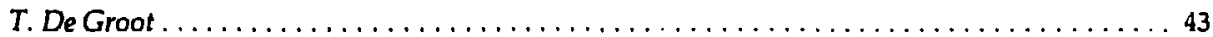

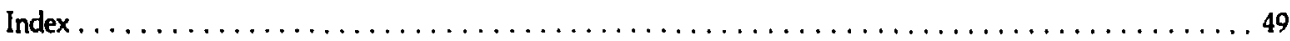




\title{
Overview-Nova Control System
}

\author{
P. Van Arsdall
}

Nova is a large, pulsed-laser facility that supports Inertial Confinement Fusion (ICF) research $^{1}$ at Lawrence Livermore National Laboratory (Fig. 1). The latest in a series of increasingly energetic neodymium-glass laser systems at Livermore, Nova his ten laser beams, each with a path length of $25 \mathrm{~J} \mathrm{~m}$ and an output diameter of $74 \mathrm{~cm}$, that can concentrate 100-150 TW of optical power for periods from 100 ps 103 ns onto an experimental fusion target. The resulting physics data are studied to better understand the proces'es involved in ICF. Inserted just prior to the fina. focusing lens at the target chamber, potassium dihydrogen phosphate (KDP) crystal arrays in the beam path either double or triple the frequency of the fundamental $1.06-\mu \mathrm{m}$ light for irradiation experiments at any of the three possible wavelengths.
Operation of a complex electrooptical-mechanical system such as Nova requires a sophisticated control system with an easily understood manmachine interface. Because the laser operating staff is unable to be both technical specialists and control system experts, this interface must be easy to master. Since the Nova ICF facility supports a robust experimental program using the latest in instruments, configurations, and methods, the control system must be particularly adaptable to change. Approximately $\mathbf{5 0 0 0}$ control and dataacquisition points are distributed over a large area, so centralized operation is necessary to effectively coordinate the target experiments.

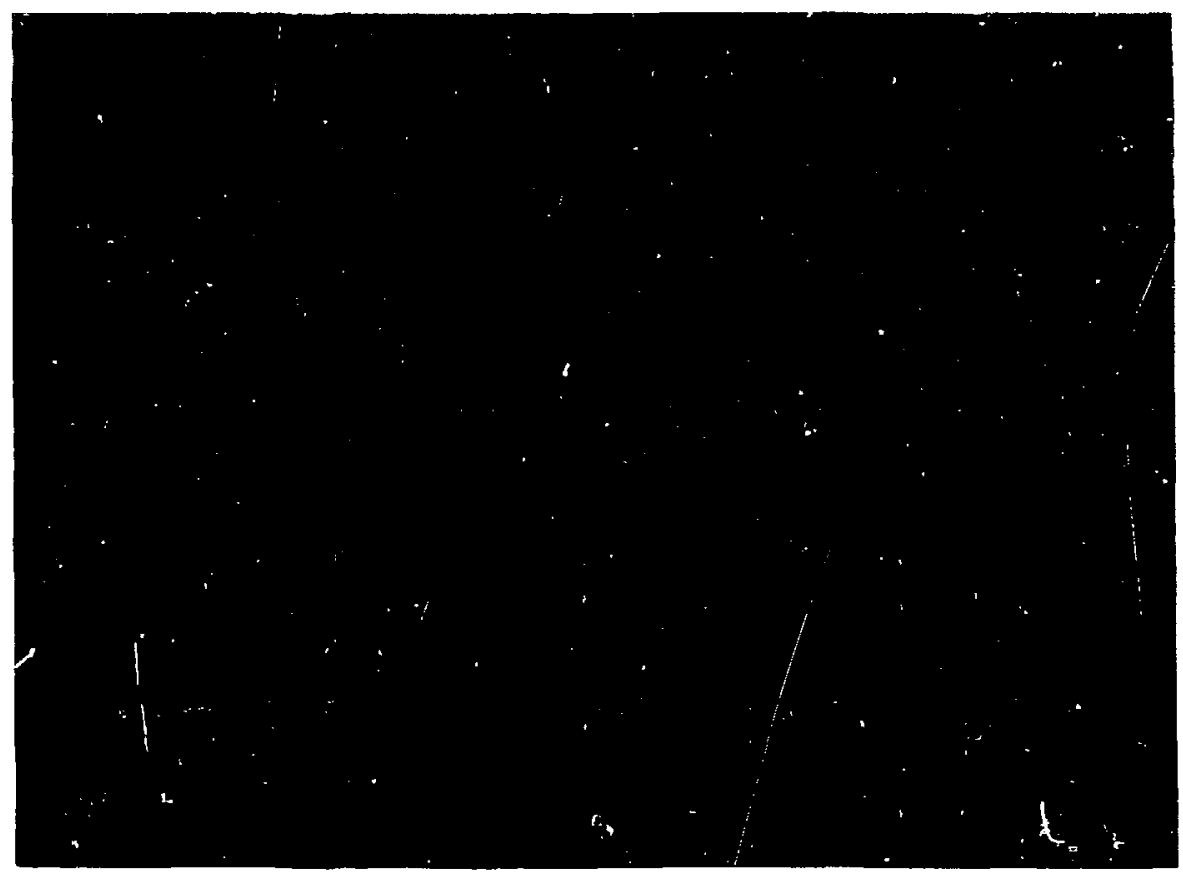

Fig. 1. Nova laser facility. 
Functionally, the control system is organized into five major parts. A central control system unifies and coordinates four subsystems in a master control room. The four subsystems correspond to major areas of the laser system. The power conditioning subsystem actually fires the laser and orchestrates much of the timing for the other subsystems. Up to $40 \mathrm{MA}$ of current flows at a force of $25 \mathrm{kV}$ during each target shot. The alignment subsystem controls the transport of the laser beams throughout the facility and the focusing of the beams on targets as small as a grain of sand. Image analysis is employed to determine alignment references from video sensors that view the laser beams. The laser diagnostic subsystem monitors the laser's optical performance and gathers data for postshot analysis. The target diagnostic subsystem records data generated by a variety of instruments designed to measure phenomena occurring during the target implosions and controls the vacuum environment of the target chamber and instruments. A unique software mechanism, the shot scheduler, assists the operators in executing the target experiments by interacting directly with the four subsystems.

The six articles of this issue describe the central controls system, each subsystem, and the shot scheduler software. Articles appear in the suggested reading order, which roughly corresponds to the order of activities necessary to perform a routine target implosion experiment. The articles may be read individually, but several subjects common to the subsystems are detailed in the article on the central controls system. The systems described in these articles have been developed through the efforts of many people on the Nova project. The principal contact listed for each article can either answer questions directly or will be able to forward requests for further information to the appropriate project personnel.

\section{Reference}

1. W. W. Simmons, R. O. Godwin, C. A. Hurley, E. P. Wallerstein, K. Whitham, J. E. Murray, E. S. Bliss, R. G. Ozarski, M. A. Summers, F. Rienecker, D. G. Gritton, F. W. Holloway, G. J. Suski, J. R. Severyn, and the Nova Engineering Team, Engineering Design of the Nova Laser Facility for Inertial Con finement Fusion, Lawrence Livermore National Laboratory, Livermore, CA, CONF-811040 (1982), compendium of 11 papers submitted to the IEEE 9th Symp. on Engr. Problems of Fusion Research, Chicago, IL. 


\title{
Centralized Computer-Based Controls of the Nova Laser Facility
}

\section{J. Krammen}

\begin{abstract}
This article introduces the overall architecture of the computer-based Nova Laser Control System and describes its basic components. Use of standard hardware and software components ensures that the system, while specialized and distributed throughout the facility, is adaptable.
\end{abstract}

\section{Introduction}

The Nova laser is controlled by a two-level distributed computer system organized as four functionally distinct subsystems and a unifying central control system. Each subsystem, described individually in other papers, controls laser-facility devices unique to that subsystem. Central Controls provides many of the basic components with which subsystems, are constructed, integrated, and interfaced to Nova operators and scientists.

Central Controls includes

- Computers and data-storage devices

- Operator consoles and related software

- Data communications hardware and software packages

- The Praxis programming language

- Data-management software

- Programming management software Systemwide use of these components promotes centralization, coordination, and adaptability of controls.

The Nova control system is highly adaptable to changes in facility requirements not only because it is distributed and computer-based, but also because it uses interchangeable computers and standardized Central Controls software. For example, most software is written in the same Praxis programming language, is executed on the same types of computers, and is maintainert with a single software-management mechanism. ${ }^{1}$ The same Central Controls components transfer data within and between diverse subsystems, providing a uniform mechanism for coordinating control functions. Operators use common consoles to communicate with all subsystems, thus resulting in uniform and centralized access to all subsystems.

\section{Control system computers}

Approximately 55 LSI-11/23 microcomputers act as distributed frontend processors (FEPs) for the VAX triplex-the trio of VAX computers that perform remote command and control operations, high-level automated control functions, and highvolume data storage and analysis (Fig. 1). The number of FEPs changes, depending on the experimental requirements. The FEPs provide localized control and data acquisition for the nearly 5000 control and data-acquisition points distributed over the entire laser facility. The VAXs exchange data with the FEPs through the Novanet and Novabus communication hardware networks.

Each VAX in the triplex has 4 megabytes of individual memory; the entire trio shares an additional 512 kilobytes of common memory. Though each VAX in the triplex usually runs specific subsystem applications, the entire control system can operate at reduced performance levels from one or two VAXs if necessary.

Other factors, in addition to the physical distance between Nova components, suppcrt the decision for distributing computers throughout the facility. ${ }^{2}$ First, a distributed computer system permits small, independeni teams of designers to develop and operate controls for functionally distinct and geographically separate subsystems. Second, because the manipulation of laser devices takes time, it is advantageous from both cost and performance perspectives to use many small, fast computers rather than a few large, centralized machines to control individual devices.

While FEPs are ideal for controlling and acquiring data from widely distributed laser devices, other operations require centralization for effective laser shot coordination. For example, memory-intensive control functions, centralized operator interaction, data analysis, and high-level automation and coordination are perforned by the VAX triplex.

While the VAX triplex supports the operation of the control system software, !wo additional VAX computers are important to the developinent and usage of the Nova control system. The first 


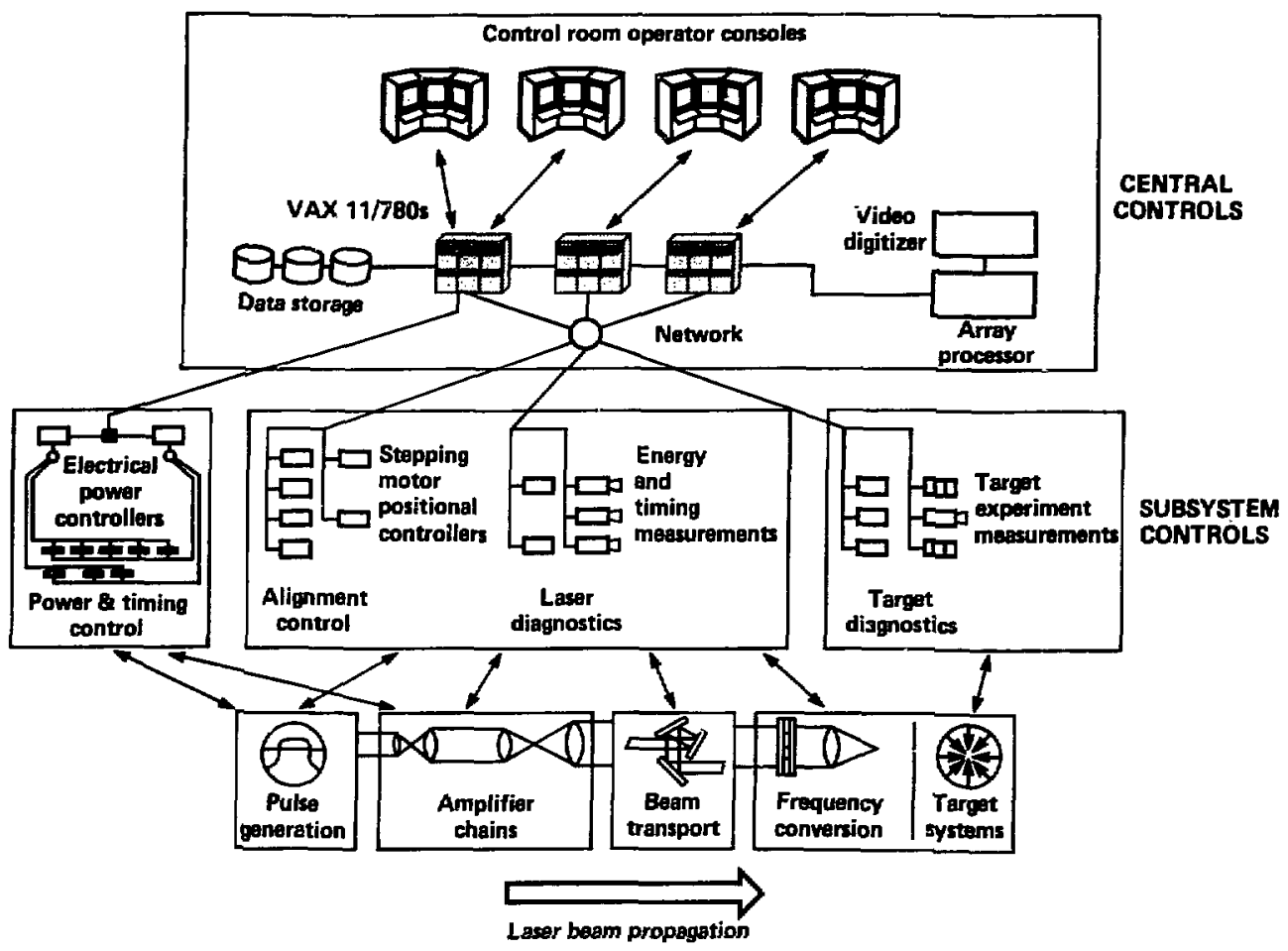

Fig. 1. Components of the Nova computer-based distributed control system. The system consists of Central Controls and four subsystems. The specialized subsystems control:

- Generating an initial laser pulse.

- Splitting and amplifying the pulse to form 10 high-power laser beams.

- Aiming and focusing the pulses onto a small target.

- Gathering data related to the performance of the laser and the target experiment.

Each subsystem consists of unique arrangements of control devices, sensors, and small computers. Central Controls coordinates subsystem functions by providing data communications and storage, video analysis, and sophisticated control functions through operator consoles and powerful computers and programs. 
manages the development, testing, and data storage of the control system hardware and software without interfering with the VAX triplex. (Software is not developed on the running VAX triplex.) The second VAX is used solely to process and analyze the physics data from each laser shot. These data are temporarily stored in the VAX triplex when generated and are then sent over a fiber-optic link to the VAX (located in a separate LLNL building) for processing and analysis.

\section{Operator console}

The control system has four identical consoles, each designed to be used by a single operator. A typical console is shown in Fig. 2. In practice, one console is used for each of the four principal subsystems; however, subsystem control functions can be combined on any single console if necessary.

Each console cabinet contains three color monitors: the center monitor for command function displays and the two side monitors for subsystem status information displays. The consoles are designed to be comfortable even when used over long periods of time. Large, flicker-free screens minimize operator fatigue. The monitors are oriented to reduce glare from the screens and make viewing easier. The center screen is touch-sensitive, thus allowing the operator to select operations from command displays by touching the desired function on the screen.

Cominand displays provicie menus of functions that allow operators to designate devices, advance through a hierarchy of function selection displays, plot data, and activate various status displays. Specialized input devices, (such as a joystick for manual, slow-slewing alignment adjustment, a numeric keyboard for data entry, and a computer terminal for online software testing) are used as an adjunct to the touch-sensitive panels. Each console includes at least one pair of black-and-white TV monitors. These monitors are connected to a network of TV cameras throughout the facility to

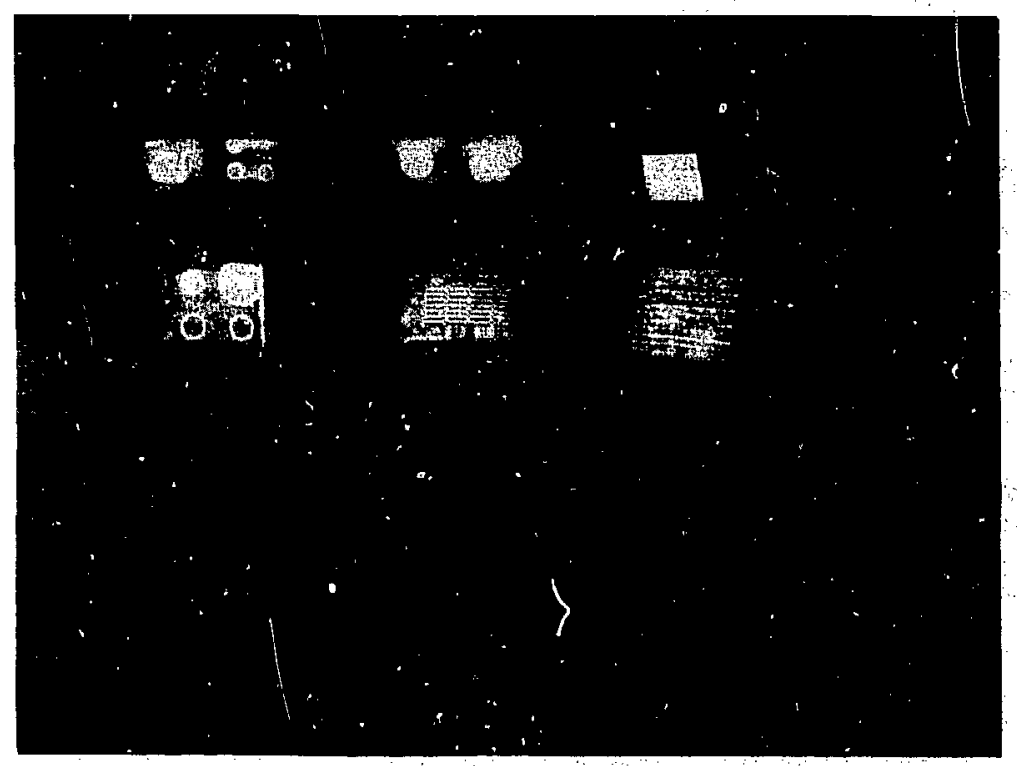

Fig. 2. Typical operator control console. Large, color console screens display laser facility status information. The center screen is touch sensitive, allowing an operator to selext control functions from a display of options by touching text and pictures on the screen. The console's computer terminal provides on-line software testing and other computer interaction capabilities. The joystick provides remote control of electromechanical laser alignment devices. Operators view laser alignment on the smaller monochrome monitors mounted at the top of each console. 
provide video images of the laser beams. The TV distribution system is discussed as part of the Nova alignment subsystem.

The color monitors of each console are attached to a graphics controller. Each graphics controller is attached to a VAX and is programmed and controlled by specialized graphics software running in each VAX. The VAXs, graphics controllers, and graphics software together graphicdliy display pictures of control system devices on the console screens. The graphics software modifies the color and text of displays based on inputs from control system programs running in the same VAX and in response to touch-panel inputs. Modifications occur when control-system programs provide data to the graphics software for processing and output to the graphics controller, which generates displays on the console screens. Touch panel data are received and processed by the graphics software and are sent to control system programs as control system function selections. Each subsystem uses its own copy of the same console graphics software.

The most important characteristics of the graphics software are speed and programmability. Operator selections are usually acknowledged within a fifth of a second, and screen updates typically lag update requests from control programs by less than half a second. Any one of over 200 predefined control and status displays can be selected and used within seconds. The graphics soiftware allows programmers to easily define command and status displays and to modify existing ones. The software also allows control programs to use control system device names rather than complicated graphical coordinates when specifying updates for the screens. Multiple control programs interact in a coordinated way with each console through the graphics software. Each display may be updated by a single program or by many programs, and many displays may be updated by a single program. When operators advance from one command display to another, teuch panel inputs from the new display are automatically forwarded as appropriate.

The graphics software is augmented by a vendor-supplied, general-purpose plotting software package. ${ }^{3}$ The plotting package is very programmable. This powerful package plots graphs of preand post-shot data on the console screens and on off-line graphics plotters and displays. For this application, flexibility is more important than speed. Programs that generate very readable plots in almost any style are easily written. However, complicated plots sometimes take minutes to draw.

\section{Data communications hardware/ software packages}

The shared memory, Novanet, and Novabus packages described below communicate shot, command, status, and synchronization data between computers and the control system devices and software packages. These software and hardware packages were specifically designed to provide fast and reliable communication without heavily loading the computers.

\section{Shared memory}

Some control system computers share portions of main memory. Shared memory is particularly well suited to providing identical status information to many processes in many computers. The three VAXs share 512000 bytes of memory for communication between programs on different VAXs. Figure 3 shows how programs within the VAXs share memory. Commurication is initiated when a program in one computer stores data in areas of memory directly readable by other coniputers. Programs in computers sharing memory then access the data by reading it from memory. Computer time is conserved because processes waiting for data upciates can be suspended until the updates occur. A similar technique provides communication between programs in the same VAX computer, where a section of each computer's main memory is set aside for concurrent use by more than one program.

The Nova Shared Memory (NSM) package provides synchronization between those programs that use shared memory communication. NSM software organizes data within shared memory into groups called shared data tables. Each table has a unique name. When a program first accesses a table, the program invokes an NSM function that "associates" the table with the program. Each program using a table then invokes other NSM functions during each access. For example, when a program reads data from a table, an NSM function notifies the program each time new data is available in the table. Also, before a program must write data, another function reserves the table for that program. When the data are written, another function notifies programs reading the table that an update has occurred. Finally, the NSM software package includes an "analyzer" utility to assist in diagnosing communications activity in shared memory. All subsystems use the NSM package and shared memory to communicate data and to coordinate control functions. 


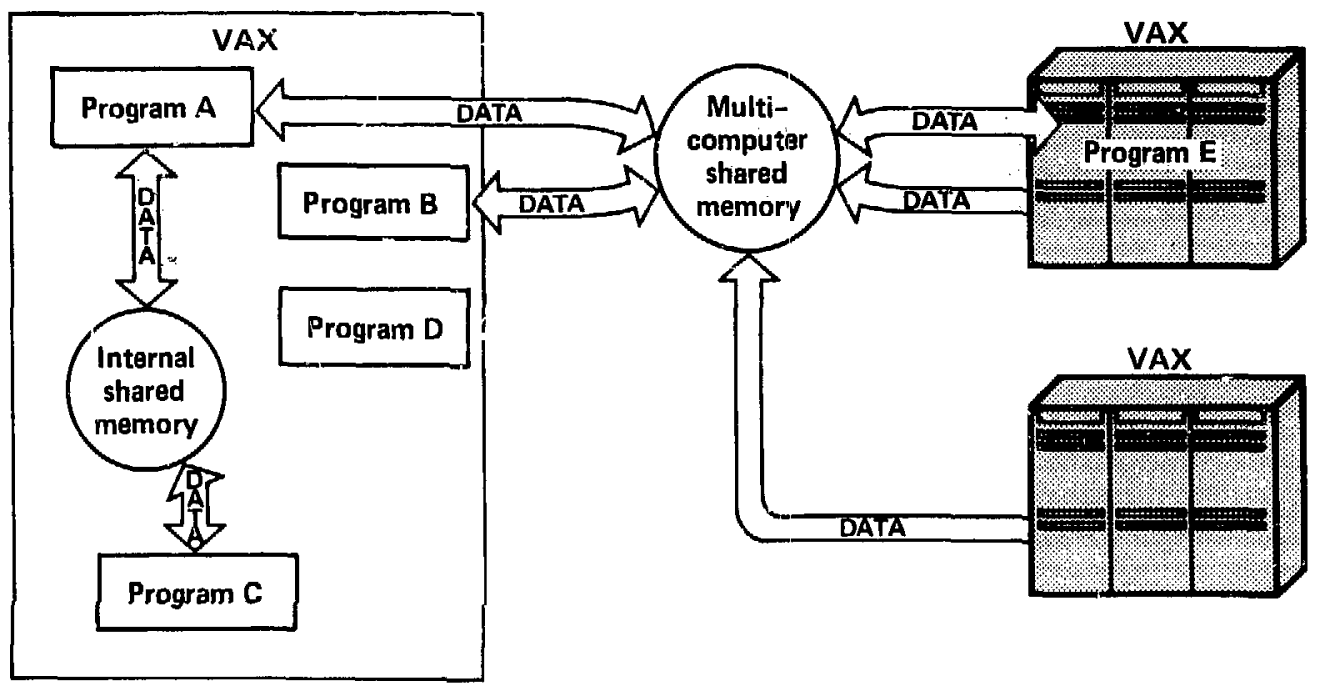

Fig. 3. Memory-sharing scheme for programs within and between VAXs. Most programs, (such as Program D) use memory not shared by any other program, called local memory. However, some programs in the same computer (stuch as $A$ and $C$ ) share portions of memory with each other or with programs in other computers (such as A, B and E). With proper interlocking, information is easily passed between programs throught shared memory because data written by one program can be read by one or more other programs.

\section{Novanet}

Novanet is a packaze of hardware and software components specifically designed to provide communication between Nova control system VAXs and FEPs. ${ }^{4,5}$ The package al o provides for data transfers directly between curtain control system devices and the VAX romputers. Novanet connects over $50 \mathrm{FEPs}$ and 20 special control system devices to the 3 VAXs. The distance between components connected with Novanet can vary from 20 to $200 \mathrm{~m}$.

Novanet is schematically shown in Fig. 4. Each VAX and FEP contains a Novanet hardware inte.face called a master/slave controller (MSC) board. Each VAX and FEP also contains specialized software to send and receive data through the MSC. Two fiber-optic cables attach each MSC board to the Node Star Novanet hardware, which acts as a distribution center for all data transmitted on the fiber-optic cables. Every computer and device that uses Novanet is connected to the Node Star.

Typically, VAX programs manipulate laser facility devices by communicating through Novanet with FEPs interfaced to the devices. Howevcr, some devices are connected directly to VAXs because, for these devices, the special programming and grouping provided by FEPs is not required or is not as important as fast response and reduced cost. The $Q$-bus device interface (QBDI) and the memory device interface (MDI) are the two types of Novanet hardware used for direct device communication.

Novanet transfers data very quickly and reliably. Up to 320000 usable data bits can be transmitted per second. Data are transmitted in a "packet" containing a data word combined with additional information. Novanet hardware interfaces automatically gen'rate a packet for each transmission. The receiving interface uses some packet information to determine if data were transmitted without error. The source and destination of each transmission are also included in the packet. Receiving interfaces use this information to determine whether to ignore or to accept and automatically ackriowledge each packet. These error detection and data routing functions are performed by hardware rather than by software to increase speed and to conserve computer processing resources. 


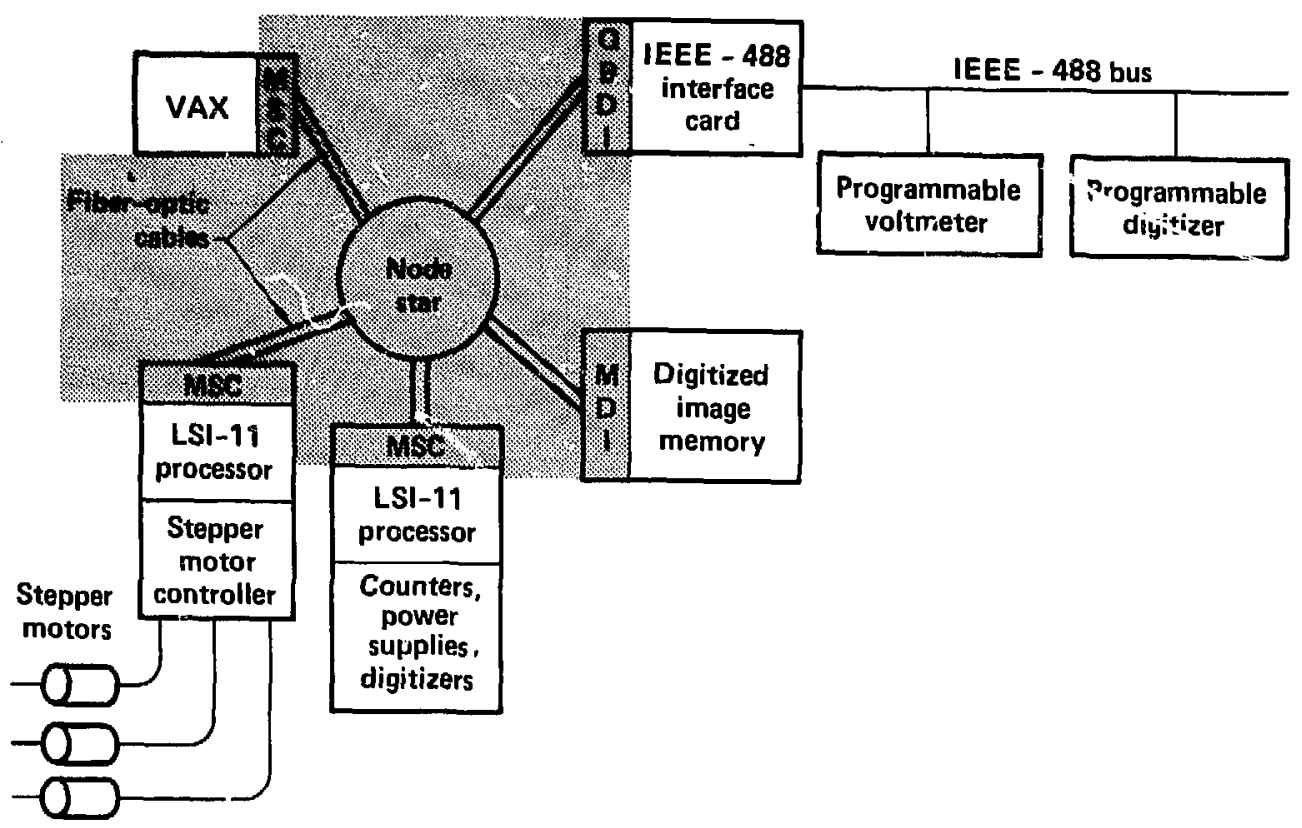

Fig. 4. Schematic representation of Novanet, showing a variety of devices controlled. Computers communicate with the network through master/slave controller (MSC) interface hardware. Laser control devices, such as stepping motor controllers and electronic sensor controllers, are attached to LSI-11 computers. Some applications require $Q$-bus device interface (QBDI) and memory device interface (MDI) hardware, which attach laser control devices and sensors directly to the network. The Novanet Node Star hardware distributes all transmitted data.

Fiber-optic cables further enhance reliability. Fiber optics, by transmitting light rather than electrical signals, are immune to electrical noise and can handle fast data transfers. Also, connected computers and devices are electrically isolated from high voltages over the length of the cables.

\section{Novabus}

Novabus is the communication network through which devices in the power-conditioning subsystem are attached to the FEPs. The network also distributes timing signals generated by the power-conditioning subsystem to devices in other subsystems. Novabus provides a communication mechanism for many tasks that require relatively simpie device control functions but that must be closely synchronized during a laser shot.

Novabus provides simple device control and synchronization through its unique method of communicating data. Novabus hardware is designed to duplicate the fundamental hardware communication signals used within the FEP. Device control hardware is "plugged" into the Novabus network just as it would be plugged into an FEP. Modifying the state of a device on the Novabus network is accomplished by simply updating a memory location in the FEP. FEP programs thus read and write digital control memory within remote power conditioning devices as though it were computer memory. 
Novabus uses the same fiber-optic technology as Novanet. Fiber-optic cables provide electrical isolation between the power conditioning computers and the high-voltage devices they control. The Novabus hardware components include two fibtr interface controllers, which connect the dual power conditioning LSI-11 FEPs to fiber-optic distribution hardware, and fiber interfaces for connecting each group of physically local devices to a fiber (Fig. 5). Each fiber interface relays the signals on the incoming fiber to an outgoing fiber connected to the next interfare, resulting in chains of fiber interfaces. As with Novanet, the Novabus distribution hardware "broadcasts" its signals to devices on up to 16 íber chains
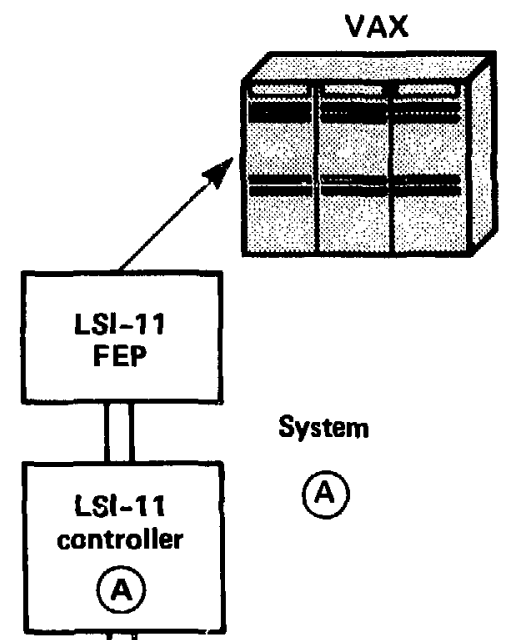

System
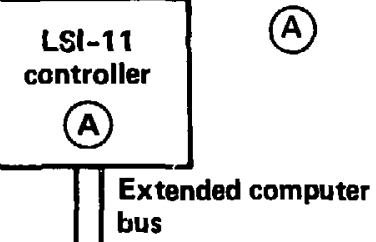

simultaneously. Each fiber interface accepts and acknowledges only those signals addressed with the proper source and destination information for that interface.

Currently, 100 groups of local devices are attached to 12 of these chains. Redundant fiber interfaces connect each local device group to two distinct Novabus fiber chains, each served by a different power conditioning FEP. The redundant communication links and FEPs increase reliability because all necessary data transfers still occur as long as one set of hardware remains operational. When both sets of hardware are working, communication functions are shared for an overall. improvement in performance.

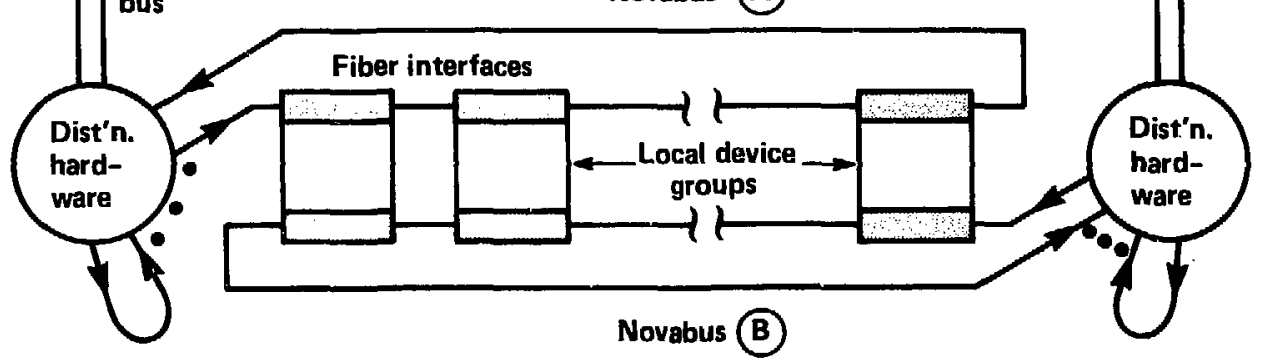

Fig. 5. Schematic representation of Novabus, showing connection of local devices to FEPs. Data from the LSI-11 are broadcast to all fiber interface chains through the controller and distribution hardware. Each fiber interface decodes transmissions and determines if the data are addressed to devices in that group. Data from devices are broadcast to all chains back to the LSI-11 computer. Redundant LSI-11s, controllers, distribution hardware and chains improve reliability. 


\section{The Praxis programming language}

Praxis is a modern, high-level computer language designed for the efficient programming of control systems. The Nova project, through the Department of Energy, has fostered the development of the Praxis language ${ }^{6}$ to achieve some essential goals of the designers of the ADA language 7 (a DOD standard implementation language) on a schedule useful to the Nova project. The language provides for development of software composed of separately compiled modules (i.e., sets of integral programming instructions), user-defined data types, and many other modern programming language mechanisms. Direct access to computer functions, efficient data manipulation at the bit levels, and interlocked data access between modules are among the Praxis language features that greatly enhance control system power and flexibility.

Praxis also contains techniques that enhance program readability and simplify the sharing of software between subsystems. Praxis supports separate compilation of program moulules and compares programmer-declared data types between dependent modules at compile time. Programming errors related to incompatible data types are found during program compilation, usually greatly reducing the time necessary to debug software once it is successfully compiled. Praxis also allows programs to be written at varying levels of detail: programs can be written for more than one type of computer, or programs can use instructions and data formats specific to a certain computer or device, as necessary. This feature also makes possible more efficient programming.

The Praxis compiler, written in Praxis, executes under the VAX system and generates optimized code for VAXs, PDP-11s, and LSI-11s. Approximately $80 \%$ of software in the control system is written in the Praxis language.

\section{Software management}

The Nova Software Quality Assurance Plan ${ }^{8}$ establishes specific programming techniques and administrative controls that form the basis for Nova software management. Of more than 126 man-years invested in the Nova control system, approximately $60 \%$ have been spent exclusively on software development. Over 300000 program lines and thousands of lines of computer-stored program documentation are currently maintained in the system.
Software is written and maintained on a VAX computer separate from the triplex to isolate development from normal control system operation. Programs are developed and maintained as packages called "products." A software product may consist of either one program or hundreds of programs that, as a group, perform a single logical function within the control system. Software specific to each control subsystem is an example of a product. A software product may consist of many smaller subproducts. Programs in a product often invoke programs from another product. Utility programs within the VAX operating system maintain product boundaries and provide controlled access to products.

Three complete copies of all programs and data in a product are kept in logically separate disk storage areas, or "levels." Every product is maintained at three levels of readiness, as shrown in Fig. 6. Major modifications to products and simple tests are performed at the lowest, or development (DEV), level. A product ready to be tested by programmers and laser operators in conjunction with other products is copied to the library (LIB) level. Minor programming errors may be corrected in the LIB level product, while major modifications can again be made at the DEV level. Very reliable products are copied to the system (SYS) level. By developing software products in distinct levels, operators have reliable softwaie, while programmers are able to implement and test new products.

Standards have been developed for documentation within programs, for organization of programs within a product, for names of procedures for recompiling and regenerating a product, and for access to a product. Peer reviews maintain adherence to these software packaging standards. Administrative procedures are used for coordination of updates, for on-line testing, and for activation of software products.

\section{Task definitions and data management}

Definitions for control-system tasks for daily experimental operations or maintenance are stored in computer parameter files maintained separately from the programs. Definitions are made for groups of related control devices, such as laser components and their interdependencies, target and laser diagnostic instrument calibrations and range settings, and sequences of control operations. Changes to these definitions are made by modifying the data 


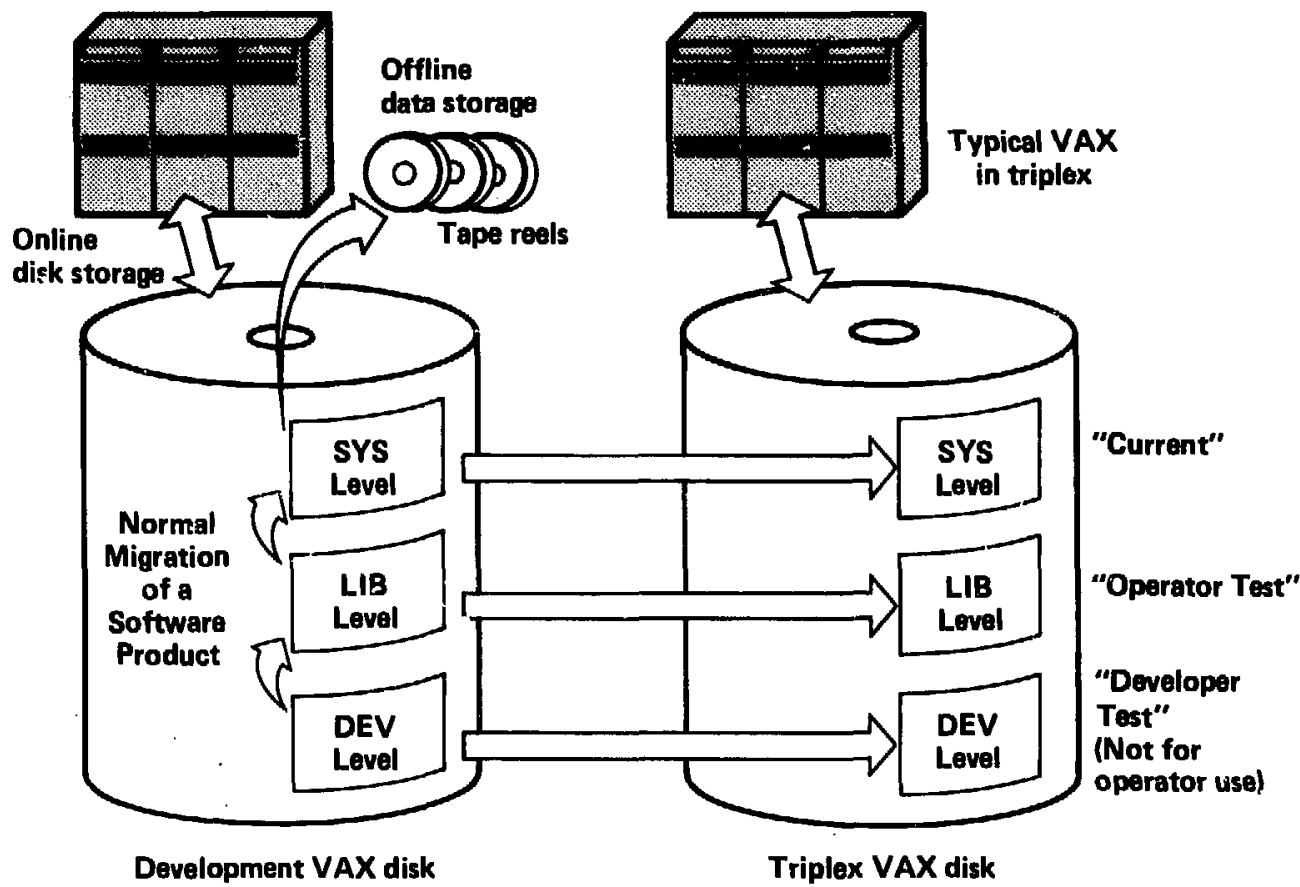

Fig. 6. Software-development hierarchy used for program management. Software is grouped into "products." Each product, consisting of up to hundreds of programs, is maintained at three levels of readiness in all VAX computers in reserved areas of the storage disks. Product software is copied from development (DEV) level to library (LIB) and then to system (SYS) level as testing and modification improve reliability. As a version of a product becomes more refined, all other versions are displaced to the next level or to offline storage. Because programs are not developed on the triplex VAXs, only "runnable" programs are copied from development computer levels to VAX triplex disks.

files rather than by reprogramming, recompiling, and retesting the computer software. The definitions can be examined and changed by operators rather than by the software specialists who designed the system.

Raw and partially processed laser and target performance data are combined with relevant control system configuration data on a subsystem basis to produce "shot data." These data are transmitted through a fiber-optic link to another VAX$11 / 780$ computer dedicated to target data analysis. Laser configuration and performance summaries are maintained in Oracle, a vendor-supplied, relational data base system on the software development computer. ${ }^{8}$

\section{Conclusion}

We have standardized and centralized some resources in the distributed Nova control system to make it easier to adapt, coordinate, and operate the system. Distributing computer resources allows small teams of semi-independent developers to efficiently construct and maintain specialized subsystems. Standardized computer hardware, softwaremanagement techniques, data communication components, programming language, and operator consoles promote the construction of an adaptable and specialized system of distributed computers. 


\section{References}

1. F. W. Holloway, Strategies of Design, Development and Activation of the Nova Control System, Lavirence Livermore National Laboratory, Livermore, CA, UCRL-89552 (1983), presented at the 10th Symposium on Fusion Engineering, Philadelphia, PA.

2. G. J. Suski, J. M. Duffy, D. G. Gritton, F. W. Holloway, J. E. Krammen, R. G. Ozarski, J. R. Severyn, and P. J. Van Arsdall, The Nova Control System-Goals, Architecture, and System Design, Jawrence Livermore National Laboratory, Livermore, CA, UCRL-86827 (1982), presented at the IFAC Workshop on Distributed Computer Control Systems, Tallinn, USSR.

3. DISSPLA User's Manual (Integrated Software Systems Corporation, San Diego, 1978).

4. J. R. Hill, J. R. Severyn, and P. J. Van Arsdall, "Novanet Communications Network for a Control System," Proc. 8th Data Communications Symp., Mass., 1983 (Cape Cod, MA, 1983).
5. J. R. Severyn and P. J. Van Arsdall, "Fiber Optics Provide Distributed Communications Link for Nova Laser," Electronics Engineering Department Quarterly Report, No. 2, 1980, Lawrence Livermore National Laboratory, Livermore, CA, UCRL-50025-80-2 (1980).

6. A. Evans, Jr., C. R. Morgan, J. R. Greenwood, M. C. Zarnstorff, G. J. Williams, E. A. Killian, J. H. Walker, and J. M. Duffy, Praxis Langisage Reference Manual, Lawrence Livermore National Laboratory, Livermore, Calif., UCRL-15331 (1981).

7. ADA Language Reference Manual (ADA Joint Program Office, MIL-STD 1815, Washington, DC, 1980).

8. F. W. Holloway, Nova Project Subordinate Assurance Program :Jan, Control System Software, Lawrence Livermore National Laboratory, Livermore, CA, Nova Program Quality Assurance No. M-078-10-60A (1983).

9. Oracle User's Manual (Oracle Corporation, Menlo Park, Calif. 1984). 


\section{Nova Pulse-Power Control System}

\section{J. Smart}

A Nova shot requires up to $9 \mathrm{MA}$ of current at a force of $20 \mathrm{kV}$ in less than $1 \mathrm{~ms}$. The pulse-power, or power conditioning, control subsystem automatically selects the laser power chains or devices needed to execute the shot. The power conditioning control subsystem consists of four software and hardware layers that, taken together, issue specific device commands as needed to operate the laser. The control hierarchy permits design flexibility and ease of operation, and virtually eliminates operator errors.

\section{Introduction}

The Nova pulse-power, or "power conditioning," system regulates the hardware that fires the 150 disk amplifiers, 22 rod amplifiers, and $\mathbf{4 2}$ Faraday rotators in the Nova 10-arm laser. Seven $1.5-\mathrm{MV} \cdot \Lambda$ power supplies and nine $100-\mathrm{kV} \cdot \mathrm{A}$ power supplies charge a 60-MJ capacitor bank with more than 12000 25-kV capacitors. During a full laser shot, 114 ignitron switches switch almost $9 \mathrm{MA}$ in less than $1 \mathrm{~ms}$ and fire up to 5300 flashlamps. ${ }^{1-3}$ Power Conditioning also provides timing triggers for synchronizing laser components and diagnostic subsystems and monitors several facility-related subsystems. These related subsystems provide safety interlocks, building security, nitrogen supply (for cooling laser components), and vacuum sensors. The system operator does not control these subsystems directly; rather, a unique set of interrelated computer programs automatically performs numerous lower-level functions. This paper will describe the control-system architecture and the functions of each control layer.

\section{Operator controls}

The operator controls the system from a console with three color CRT displays equipped with touch panels. Normally, the operator directs the power-conditioning system by touching any of the pulse-power, system-timing, or facility menus. The pulse-power menu, shown in Fig. 1, allows an operator to select a variety of laser components, groups of components, or laser arms by touching pictures of the desired components on the screen. The component's color reflects its status. Once operators choose a configuration, they may select the desired pulse-power sequence. The system- timing menu allows the operator to directly conitrol timing sequences as well as to select various timing iriggers and rates. The facility menu, by displayın: a map of the Nova building, allows the operator to quickly determine the condition of all facility components in a particular room or area.

An auxiliary-control program, run on a DEC VT125 graphics terminal, allows direct access to each hardware device in the system and performs low-level control and maintenance operations. Unlike programs on the console displays, this program may be easily accessed from any terminal throughout the laser facility with the correct privileges and is particularly useful during activation and maintenance. Each menu displays device status in real time and is keypad driven. A typical menu is shown in Fig. 2. A color monitor attached to the terminal will display a power-supply histogram or flashlamp-current waveform plo!.

In addition to the auxiliary-control program, utility programs are available that allow various parameter and configuration files to be loaded and saved. Diagnostic programs also allow direct operation of specific laser devices, such as individual power supplies, rather than higher-level components.

\section{Control architecture}

Figure 3 shows how three VAX-11/780 computers monitor and control the power-conditioning system. The pulse-power subsystem generally uses only one of these computers at any instant; the remaining two are reserved for the alignment and diagnostics subsystems. An MA780 shared memory and an Ethernet communications network permit communication between VAXs. 
The VAX rusponds to operator requests by generating a series of commands to the hardware devices. These commands are sent through a shared memory to four LSI-11/23 microcomputers acting as front-end processors (FEPs). The FEPs format data and interpret the VAX commands to operate the hardware devices. The FEPs also constantly poll the hardware devices for status changes and place the new information into the shared memory. The VAX monitors this information and changes control sequences or notifies the operator, via one of the display menus, as necessary.
Each FEP controls a specific portion of the power-conditioning system. One FEP normally controls the various pulse-power devices, such as the high-voltage power supplies and capacitor-bank interfaces. A second FEP times and synchronizes the system by providing numerous triggering pulses to various other subsystems and hardware devices. The third FEP normally monitors safety interlocks, security inputs, and various vacuum and nitrogen control sensors. The remaining FEP controls the fast-timing hardware that triggers numerous laser components.

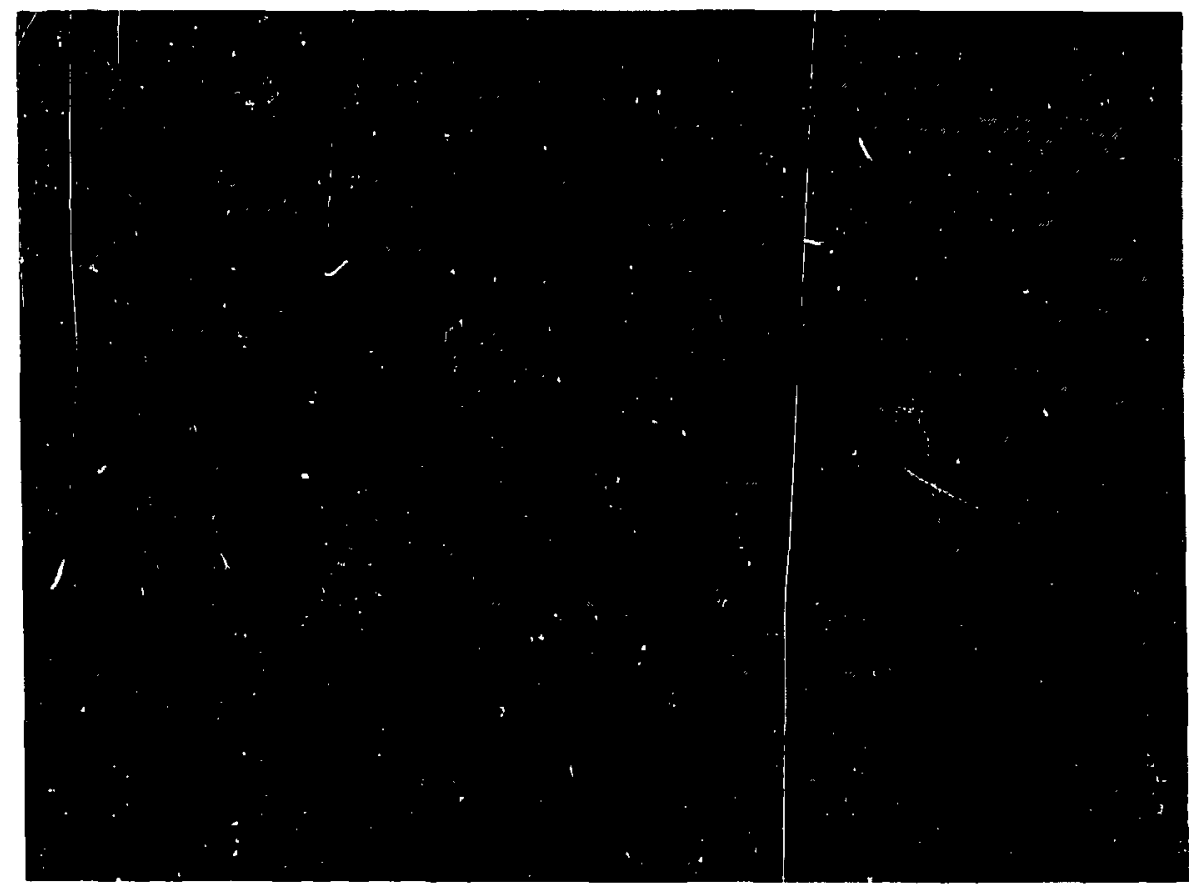

Fig. 1. Nova pulse-power menu. The menu, displayed in color, allows the operator to select a variety of laser components, groups of components, or laser arms by touching pictures of the desired components on the screen. The four basic sequencing operations defined in the virtual-control layer can also be accessed from this menu. 
The FEPs communicate with each controlled and monitored device over the Novabus interconnection network. Each FEP's internal bus is serialized for connection by a fiber-optic bus to devices throughout the laser facility. (A fiber-optic chain is necessary to provide optimum voltage isolation.) Bus messages, each containing a unique device address, originate from each FEP and are relayed through the fiber-optic chains for examination by the devices. The designated device responds by ac- cepting the command or by returning status data.

To improve reliability, each device is served by two redundant buses, as shown in Fig. 4. Identical commands are simultaneously sent from the FEPs through both buses. Either bus can perform all control and monitoring required. In the event of a device failure, messages are automatically rerouted to the alternate bus to allow other devices on the bus chain to continue operating.

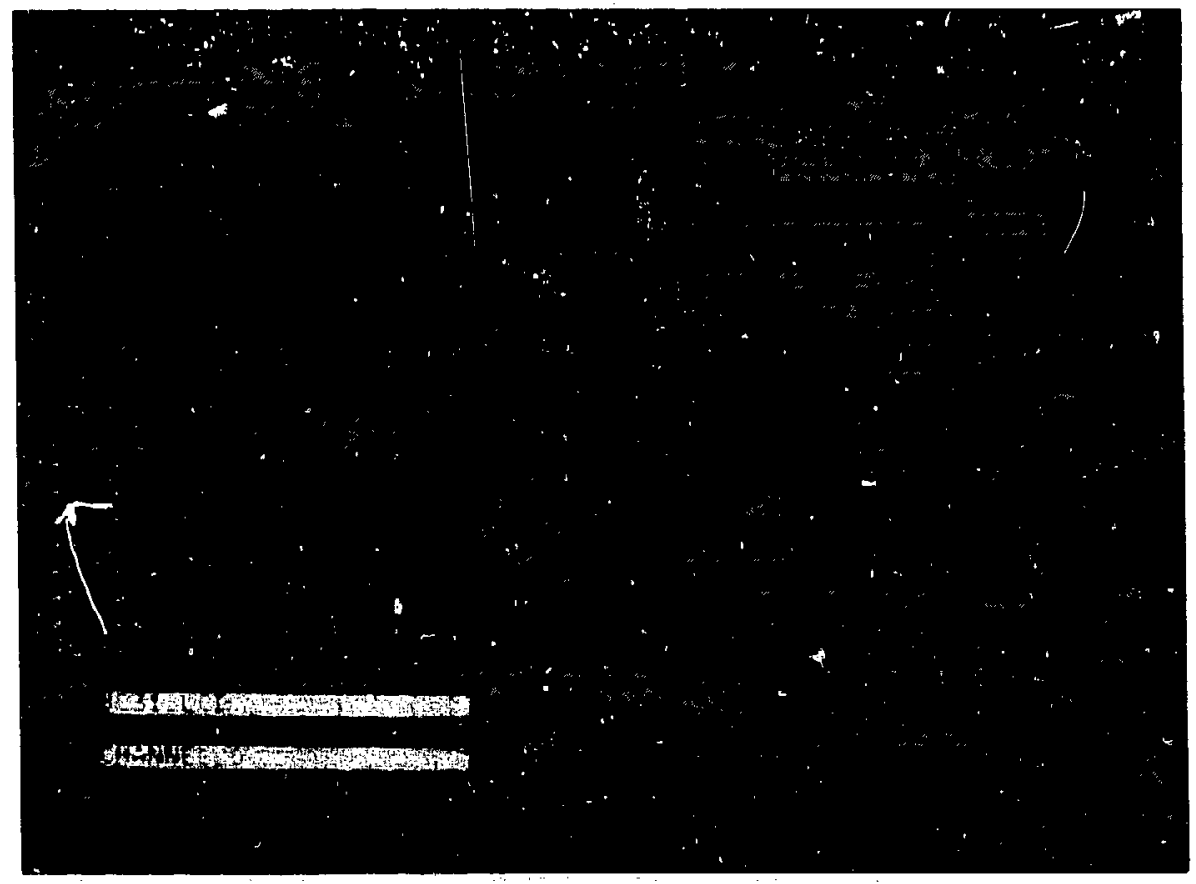

Fig. 2. Lamp circuit diagnostic program menu. Such auxiliary-control programs can be accessed from any terminal within the bser facility. Each auxiliary program menu permits direct access to hardware devices in the system. 


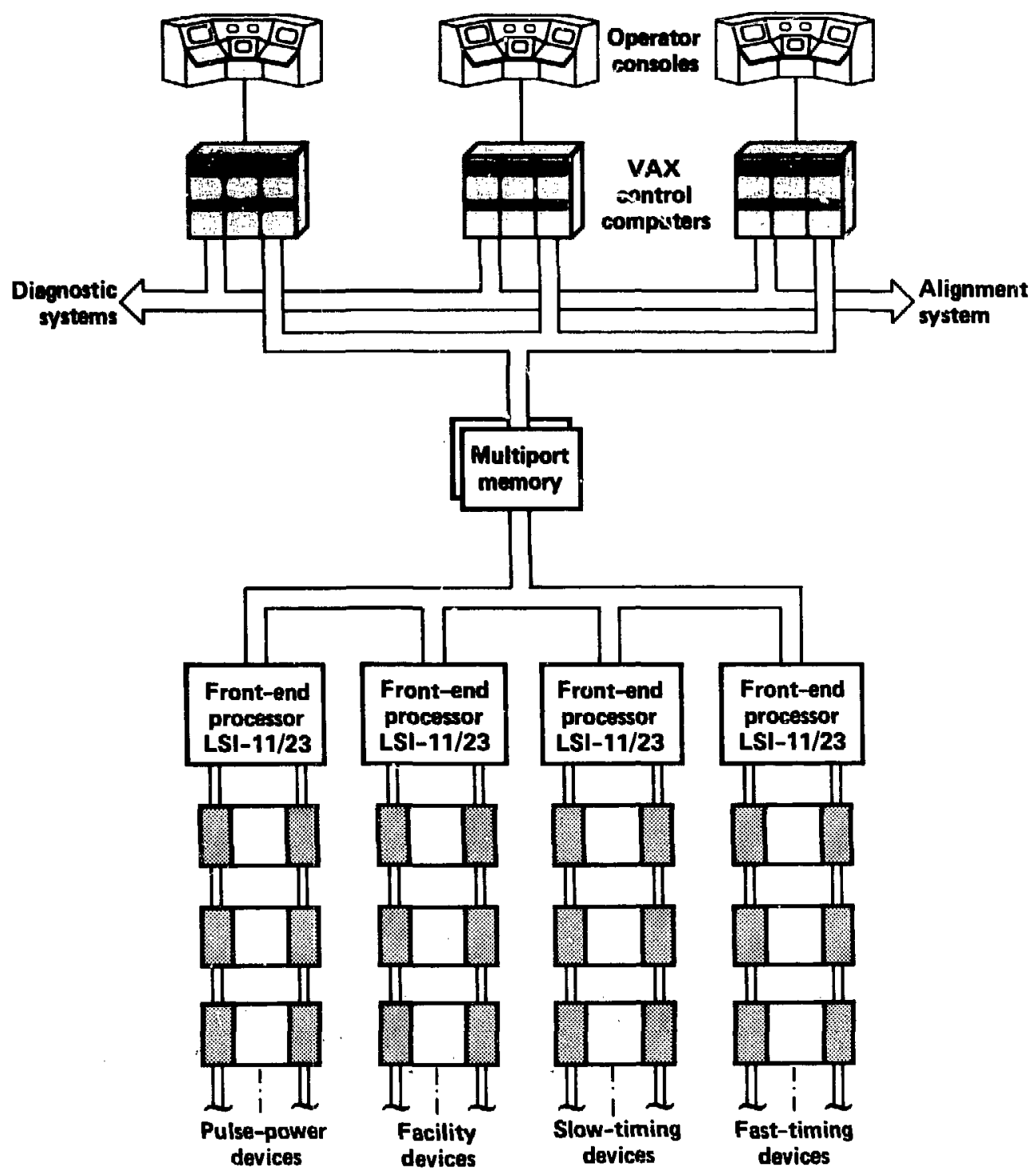

Fig. 3. Cocrdination and control of the posver-conditioning, alignment, and diagnostic systems. The frontend processors interpret commands from three $\mathrm{VAX}-11 / 780$ computers and format data to operate the hardware devices. The shaded sides of the devices represent connections to the Novabus interconnection network. 


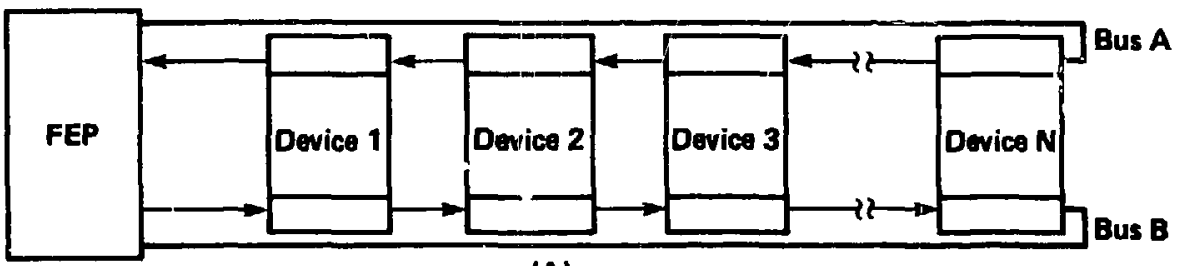

(A)

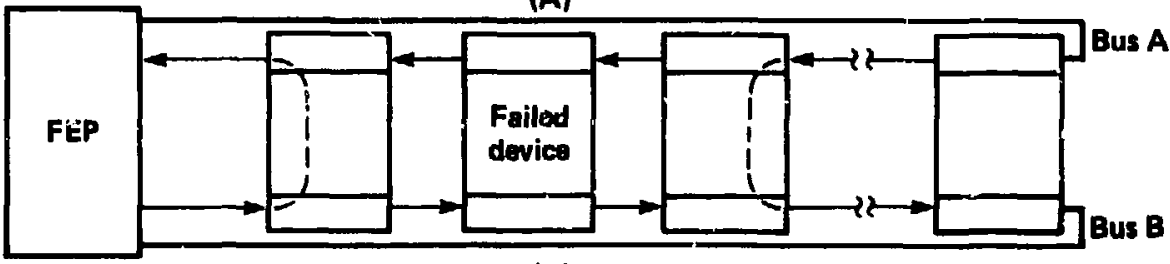

(B)

Fig. 4. Detall of redundant buses between: FEPs and laser devices. The redundant links shown in (A) improve communication reliability. If necessary, commands can be rerouted around a failed device, as shown in (B).

\section{Controls model}

A controls model, illustrated in Fig. 5, shows how the Nova control system is organized and how the system can accept a general command from the operator and translate the command into a specific action. This model provides a useful means for picturing the organization of the software and hardware hierarchies. The model divides the control system into five layers:

- System control

- Subsystem control

- Virtual control

- Logical control

- Physical control

The systent-control layer directs the entire laser, while the remaining four layers compose the power-conditioning subsystem. Each layer uses its own services to build upon the services provided by the layer below in order to provide higher-level services to the layer above. System operators work directly with the three highest layers; the lowest layer interfaces directly with the hardware control elements. Only adjacent layers communicate, and then only through strict, well-defined interfaces.

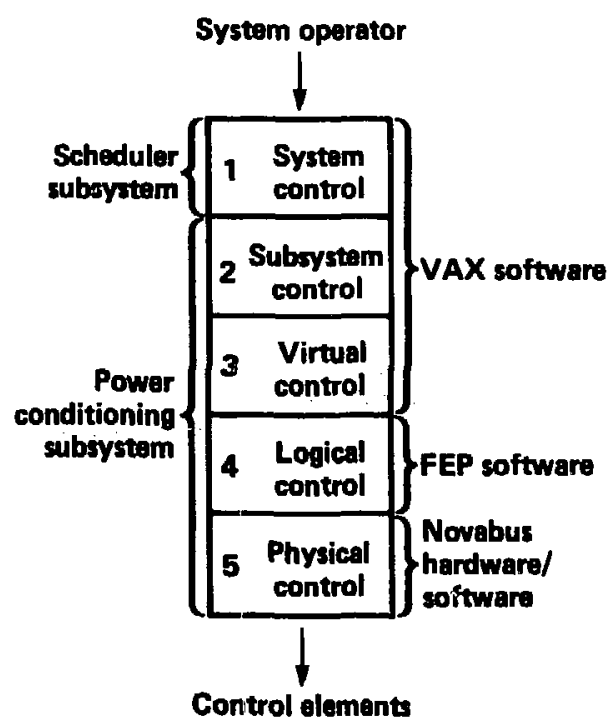

Fig. 5. Nova contuols model. The model divides the Nova control system into flve layers, each of which commands specific subsystems and hardware of software devices. 
Table 1 outlines the functions of each layer and the components or devices controlled. Each of these layers is dixussed individually in the sections below.

\section{System-control layer}

System control integrates the power-conditioning, alignment, and diagnostic subsystems into one unified system and provides system-level operator control. Althougl, the system-contro! layer is primarily composed of the stiot schedulor and the integration subsystem - both of which are discussed separately in this issue-system control uses many of the services that power conditioning provides, including sequencer control and the system clock.

The interface between each subsystem and the shot scheduler is the integration environment, consisting of predefined data structures stored in the VAX-MA780 shared memory. These data structures contain either status information maintained by the appropriate subsystem or commands for each subsystem to execute.

\section{Subsystem-control layer}

The subsystem-control layer integrates the many independent control functions provided by the lower layers into one common, unified subsystem. Whereas system control coordinates power conditioning with other layers to operate the entire laser, subsystem control specifically commands the power-conditioning subsystem. Based on the services from the layers below, this layer internally makes decisions that globally affect the entire subsystem. In addition, subsystem control provides a set of services to the system-control layer for use in systemwide operation. These services allow the subsystem to be manipulated without making internal changes.

When the nperator orders a shot, the shot scheduler commands power conditioning through the subsystem-control layer. The subsystem layer interprets these commands and selects the appropriate shot configuration. The subsystem layer then notifies the virtual layer below that a shot is to be fired and issues commands to the virtual layer based on the selected shot configuration. After the subsystem layer completes these tasks and verifies that the subsystem is ready, it accepts commands to control the sequence. As a sequence progresses, new status commands, such as the shot time and sequencer state, are placed in the integration environment for the scheduler and other subsystems to access.

Tabli 1. Layers of the Nova control system.

\begin{tabular}{|c|c|c|}
\hline Layer & Main functions and areas controlled & $\begin{array}{l}\text { Subsystems, components } \\
\text { or devices controlled }\end{array}$ \\
\hline System control & $\begin{array}{l}\text { System integration } \\
\text { Shot scheduler }\end{array}$ & $\begin{array}{l}\text { Power-conditioning subsystem } \\
\text { Aligument subsystem } \\
\text { Diagnostic subsystems }\end{array}$ \\
\hline Subsystem control & $\begin{array}{l}\text { Power-conditioning subsystem-decisions made here globally affect the power- } \\
\text { conditioning subsystem and provide services to the system-control layer. } \\
\text { Subsystam control selects appropriate shot configuration when system operator } \\
\text { orders a shot. }\end{array}$ & Virtual-control layer \\
\hline Virtual control & $\begin{array}{l}\text { Laser components-permits automation of device and component control, and } \\
\text { issues specific commands for components. }\end{array}$ & $\begin{array}{l}\text { Amplifiers } \\
\text { Faraday rotators }\end{array}$ \\
\hline Logical control & $\begin{array}{l}\text { Power-conditioning devices-translates commands from virtual control into } \\
\text { specific device commands. }\end{array}$ & $\begin{array}{l}\text { Interfaces } \\
\text { Power supplies } \\
\text { Ignitron switches } \\
\text { Oscillator controls } \\
\text { Diagnostic circuits }\end{array}$ \\
\hline Physical control & $\begin{array}{l}\text { Power-conditioning (Novabus) modules-hardware devices that execute specific } \\
\text { commands given to them by logical control. }\end{array}$ & $\begin{array}{l}\text { Input boards } \\
\text { Outpul boards } \\
\text { Analog-to-digital boards } \\
\text { Timer boards } \\
\text { Novabus converters } \\
\text { Novabus repeaters }\end{array}$ \\
\hline
\end{tabular}


Virtual-control layer

Upon selection of the appropriate shot configuration by subsystem control, the virtualcontrol layer chooses the high-level system components-such as amplifiers and Faraday rotatorsneeded for the required configuration. The virtualcontrol layer controls high-level system components and selects the low-level devices, such as power supplies, needed for a component. Virtual control then issues specific commands for each device. For power supplies, these commands may involve activation or deactivation, voltage settings, and the like. Virtual control allows for automation of many device controls by permitting the operator to work with high-level components, rather than with such numerous lower-level devices as individual power supplies and ignitrons. This layer is one of the most complex in the Nova power-conditioning system and is composed of three distinct control areas: pulse-power control, facility monitoring, and system timing and synchronization.

The pulse-power portion of the virtual layer controls high-level pulse-power components. An operator may work at the virtual level and manually select various laser components or sets of laser components for a particular sequence. Once the operator selects a configuration, the pulse-power portion of virtual control internally determines which lower-level devices are necessary, the operations that must be performed, and the times at which they must occur. As a sequence is executed, the selected hardware is automatically configured and verified. Verification failures generally result in the sequence being held, while severe failures at critical times may result in an abort.

At present, four basic types of sequencing operations have been defined: a test sequence, a pulsed-ionization lamp check (PILC) sequence, a dry-run sequence, and a shot sequence. The test sequence automatically performs several diagnostic checks and hardware self-tests to verify that the instrumentation is functioning properly. The PILC sequence then ensures proper laser-flashlamp operation without actually firing the laser. The dry-run sequence verifies that all hardware is shotready and that it will operate properly during the shot sequence. After these sequences are successfully completed, the operator may select the shot sequence that fires the laser.

The timing portion of the pulse-power virtual layer coordinates the timing and synchronization operations aciive throughout the power-conditioning system, including control of shot sequencing and maintenance of the system-countdown clock. The timing control also loads and verifies system-timing configurations. These configurations include selection of various timing triggers, along with their times (frequencies) and their rates. As a sequence is under way, the timing process continuously monitors these values to ensure that the system will trigger properly when fired.

The final portion of the pulse-power virtual layer monitors all laser-facility-related items, such as safety interlocks, security, nitrogen controls, and various vacuum-control sensors. Although the control hardware automatically ensures personnel and equipment safety, the facility control also monitors these inputs as an added precaution and as a convenient means of informing the operator of an error condition. If an error condition is detected, recovery actions, such as holding or aborting the sequence, may be automatically activated depending upon the selected operating mode.

\section{Logical-control layer}

The logical-control layer directly controls the devices that constitute each virtual-level component but does not directly control the detailed hardware interface. The logical-control layer's operation on a power supply is analogous to internal operation of a bench power supply after the operator adjusts front-panel controls. Virtual control "turns the dials," and logical control internally translates these commands into the proper hardwäre operations. On Nova, the FEPs carry out the bulk of the device control.

Requests from the VAX travel through the shared memory to the FEPs. Each FEP contains processes that service requests for each particular device type. These processes continuously monitor each device and report any status changes to the VAX; the processes also perform any necessary closed-loop control. Commands received through the multiport are formatted and sent to the appropriate device. All device monitoring and commanding is performed through the physical-level interface. Table $\mathbf{2}$ contains a list of all controlled devices.

\section{Physical-control layer}

The physical-control layer accepts commands from the logical-control software to direct the individual hardware interfaces incorporated within each device. For example, the input-output boards connected to an individual power supply perform the supply's physical control. This layer is enormous and represents a large portion of the design 
and development effort of the overall power-conditioning system.

The heart of this control layer is the Novabus described above. Several types of standard Novabus modules, such as output modules, input modulus, timers, and analog-to-digital converters, interface to the device electronics. Table 3 lists the modules presently being used on Nova:

Control electronics within each device are directly connected to the elements and sensors that drive the laser..$^{5}$ Because of this potentially harsh electrical environment, these hardware components must be designad for maximum voltage isolation and noise immunity. Devices associated with highvoltage or laser eye hazards contain seisors incorporated into the safety interlocks system. Much of this system is triply redundant to ensure fail-safe operation.

Table 2. Power-conditioning control system devices controlled by the logical-control layer.

\begin{tabular}{lc}
\hline \multicolumn{1}{c}{ Modules } & $\begin{array}{c}\text { Number in } \\
\text { system }\end{array}$ \\
\hline kV.A power supplies & 9 \\
MV-A power supplies & 7 \\
Simmer power supplies & 6 \\
Flashlamp power supplies & 6 \\
Ignitron switches & 121 \\
Capacitor-bank interfaces & 26 \\
Lamp check diagnostics & 26 \\
Interlocks interface & 7 \\
Control-system interfaces & 12 \\
Oscillator controls & 1 \\
\hline
\end{tabular}

Table 3. Power-conditioning control modules used in the physical-control layer.

\begin{tabular}{lr}
\hline \multicolumn{1}{c}{ Modules } & $\begin{array}{c}\text { Number in } \\
\text { system }\end{array}$ \\
\hline Novabus converters & 8 \\
Novabus repeaters & 178 \\
Output boards & 200 \\
Input boards & 474 \\
A/D boards & 66 \\
Timer boards & 134 \\
\hline
\end{tabular}

\section{Conclusion}

Studying the Nova control model is useful because the model shows how designers addressed the challenge of organizing many individual operations into a manageable, coherent whole. The model's flexibility also allows future designers to make additions or modifications to system parts or layers without adversely affecting the entire system. Adherence to the model has enforced structured design. The resulting system is both powerful and efficient, yet concise. Many operations, several of which were not possible in previous designs, have been heavily automated, virtually eliminating all operator errors. A large portion of the system has been operational on Nova for more than one year and has proven to be both very powerful and reliable, yet easy to operate and maintain.

\section{References}

1. K. Whitham, B. T. Merritt, R. W. Holloway, D. G. Gritton, and J. A. Oicles, "Nova Power Systems and Energy Storage," Engineering Design of the Nova Laser Facility for Inertial Confinement Fusion, Lawrence Livermore National Laboratory, Livermore, CA, CONF-811040 (1982), presented at the IEEE 9th Symp. on Engr. Problems of Fusion Research, Chicago, IL.

2. R. W. Holloway, K. Whitham, B. T. Merritt, D. G. Gritton, and J. A. Oicles, "Nova Pulse Power System Description and Status," 3rd IEEE Intemational Pulsed Power Conference (Albuquerque, NM, 1981).

3. B. T. Merritt, "Nova Power Conditioning Capacitor Bank and Power Supply Staging (10 Arrns with Additional Amplifiers)," Lawrence Livermore National Laboratory, Livermore, CA, Laser Engineering Note \#83 (1983).

4. D. G. Gritton, L. W. Berbigler, and J. A. Oicles, "Design of a Fiber Optic Multi-Tapped Computer Bus for a Pulsed Power Control System Application," 8th Symposium on Engineering Problems of Fusion Research (San Francisco, CA, 1979).

5. D. J. Christie and G. E. Dallum, "Pulse Power Circuit Diagnostics for the Nova Laser," 15th Power Modulator Symposium (Baltimore, MD, 1982). 


\section{Nova Laser Alignment Control System}

\section{P. Van Arsiall}

A computer-based control system has been developed to align the Nova laser. The system is designed to provide centralized computer controls and distributed manua! control, both integrated with automatic alignment. Computers analyze images from video sensors to locate beam references for automatic alignment.

\section{Introduction}

The alignment control system is designed to steer the 10 Nova laser beams along their 250-m paths from the master oscillator to the fusior. target. ${ }^{1,2}$ The system must be sufficiently modular to allow some sections of the laser to operate normally while others are being built or maintained. Figure 1 shows the path of one of the ten laser arms. The beam starts at the master osciliator. Mirrors senter the beam at the beginning of each laser section and point it through to the next

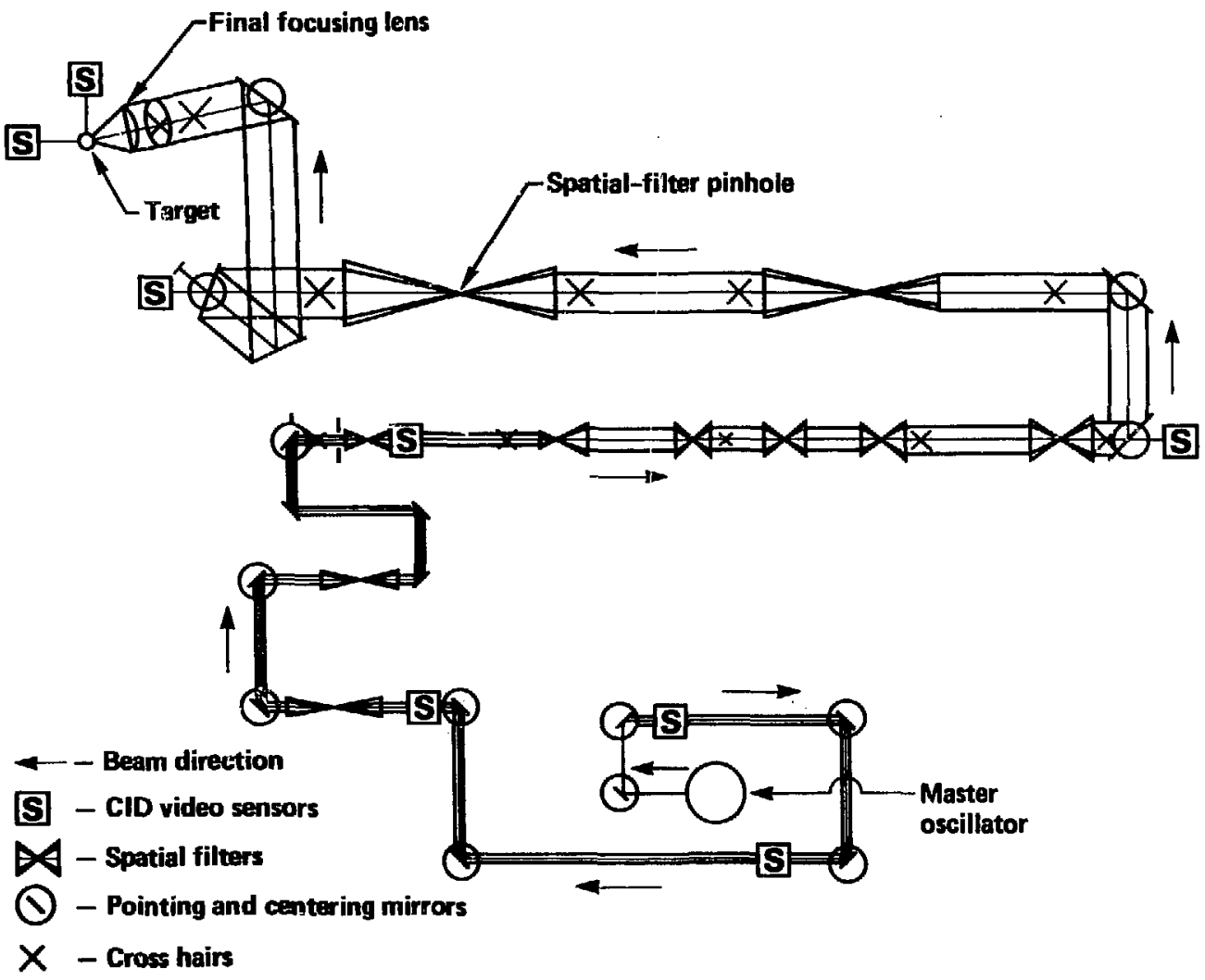

Fig. 1. Beam path of one of the ten laser arms. The beam is centered by motorized mirrors at the beginning of each laser section. Sensors sample the beam at key points along the path, and spatial filters remove destructive intensity modulation from the beam. Cross hairs are inserted along the beam path as reference points. (Beam amplifiers and other beam-path components are not shown.) 
section. Spatial filters remove destructive intensity modulation and relay the beam between laser amplifiers (not shown in the figure). A tiny pinhole in each spatial filter must be accurately positioned to allow the focused beam to pass through. Finally, the beam is aligned to the fusion target.

Figure 2 illustrates a typical alignment loop. Insertable reference cross hairs and chargeinjection-device (CID) video sensors are located along the laser path. The beam passes through a partially reflective mirror called a beam splitter, which reflects a small sample of the beam into the sensor and allows the rest to pass through to the target. The sensors take the beam sample and generate video images in a standard television format. The video images are then sent to a video switching system, which controls the input from many sensors to the digitizer. The images are digitized-translated from analog to digital format-and sent over a two-way, parallel interface to the array processor for analysis. (An array processor is a special computer designed to perform high-speed vector analysis.) The array processor sends mirror-positioning information to the VAX triplex-the trio of VAX computers that run the alignment-control software. The VAX triplex then sends commands over Novanet-a two-way, fiberoptic communications network-to the appropriate LSI-11 microcomputer to move the motorized mirrors to their proper position. When the alignment of that section of the beam is within tolerance, analysis of the beam stops and the mirrors are locked in position: the alignment loop is closed. In all, almost 300 alignment loops need to be closed by the control system to prepare the facility for a target shot. For manual alignment, analysis by the array processor is replaced by operator judgment combined with either local or remote computer control.

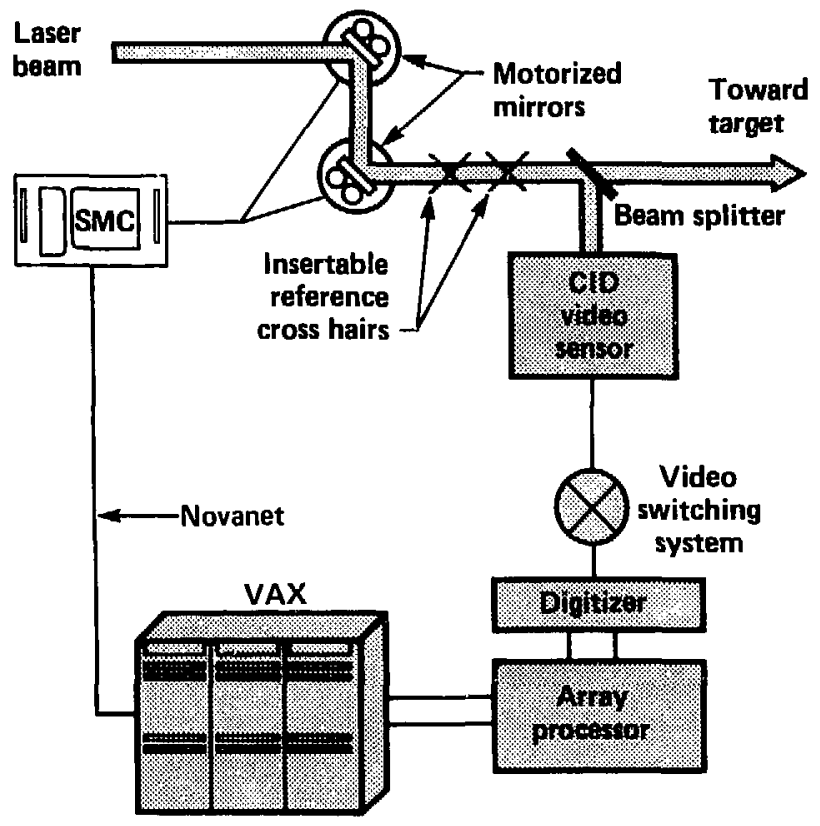

Fig. 2. Typical alignment loop. The beam is aligned by motorized mirrors. A sample of the beam is sent to a video sensor, which sends a video image to a digitizer. The digital video image is analyzed by an array processor, which sends beam-position information to the VAX triplex. The VAX then commands the appropriate LSI-11 SMC to move the mirrors. The process is repeated until the beam is aligned within tolerance. 
Operators verify alignment by observing the video images on television monitors. We have developed tests using these same images to ensure that the automatic alignment program will not misalign the laser, even under the less-favorable conditions experienced in a facility under construction.

Three major software programs are unique to the operation of the alignment system. The console alignment program (CAP) allows the operator to use manual controls, and to define which series of devices are to be moved. The CAP is also the operator's access route to the shared memory, where information from all three programs comes together. The alignment status program (ASP)

- updates the system-status displays. The automatic alignment program (AAP) aligns the laser beam without operator assistance. Figure 3 illustrates how the three programs interact through the shared memory of the VAX triplex. The interaction of the different programs is discussed in more detail in the Operator Station Controls section.

\section{Stepping motor controller}

The microcomputer-based stepping motor controllers (SMCs) are the primary alignmentcontrol building blocks. An SMC can control up to 60 stepping motors and 16 binary channels. SMC commands originate from either a local control panel or from the VAX triplex.

The SMC design is based on the alignment device-a collection of stepping motors that are related in alignment function. Stepping-motoractuated devices, which are connected to 30 LSI-11 microcomputers, are used to monitor and control the laser alignment. The ability to command groups of devices allows the operator to customize both local control panels and remote commands. Instead of fixed labels on the control-panel switches, programmable, alphanumeric displays reflect the changeable function of each switch. The operator can achieve complex configurations of many optical elements at once by programming special functions

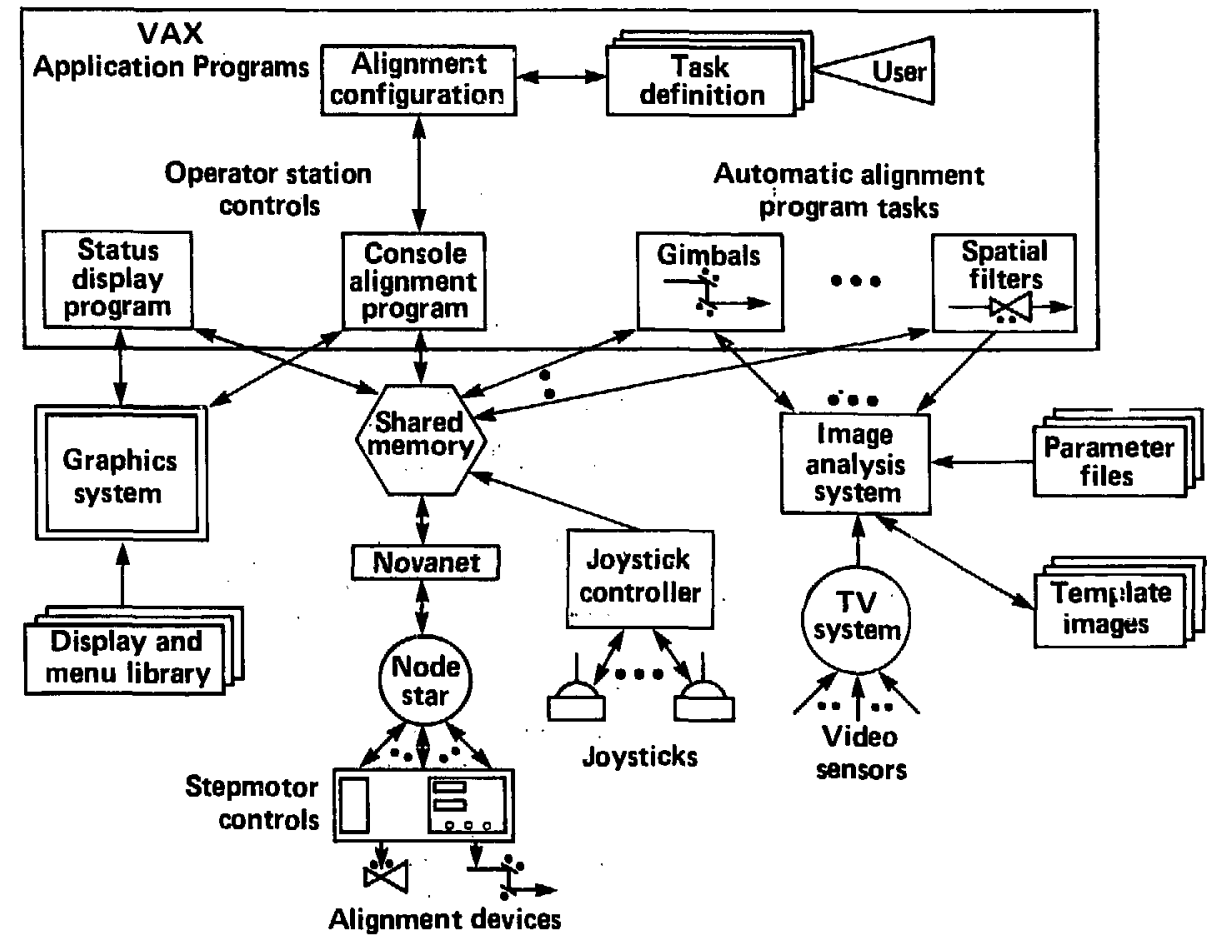

Fig. 3. Nova alignment control system, showing how the three major software programs interact through the shared memory of the VAX triplex. Two programs-the status display and console alignment programs-form the operator interface to the distributed control system. A third program, automatic alignment, performs routine tasks without operator supervision. 
to command groups of devices. An allocation system allows the operator to reserve and allocate specific devices in the same way a computer operating system allocates a disk drive or other resource. Device allocation prevents conflicts that result from two or more operators trying to use devices for dissimilar tasks at the same time.

Because of the Nova laser facility's enormous size and the difficulties we experienced with devices far from central controls, we developed special portable control panels to provide integrated controls identical to those on the SMC panel. The portable control panels permit use of integrated controls at points remote from the central computer console. Several such portable control panels may be attached to the VAX triplex through serial ports located throughout the laser facility. The portable controls allow the operator to use any alignment device in the system to support a particular maintenance job, and to observe directly the mechanical response.

The use of stepping motors in Nova requires special design considerations. First, motor power is applied only during actual positioning to reduce thermal loading of the optical environment. Second, backlash correction is necessary to remove positioning errors introduced by the mechanical hardware. Third, crosscoupling transformation from the motor coordinate system to the videosensor coordinate system is necessary because the laser path is folded and does not always lie in a plane. The transformation allows the $X-Y$ motion observed on the TV monitor to be equivalent to the $X-Y$ positioning commands.

\section{Operator station controls}

The operator station controls include both the ASP and the CAP. Operators control the distributed components of the alignment system through one of the four central control consoles. Each console has three monitor screens-the command functions appear on the center screen; the subsystem status information appears on the two side screens.

The operator accesses the CAP at the control console through a series of touch-screen menus. From the CAP, the operator can access the AAP and the ASP. The commands in the CAP are layered in a four-level hierarchy from general to specific (Fig. 4). At level one, the operator selects

Screens: Left Center Right

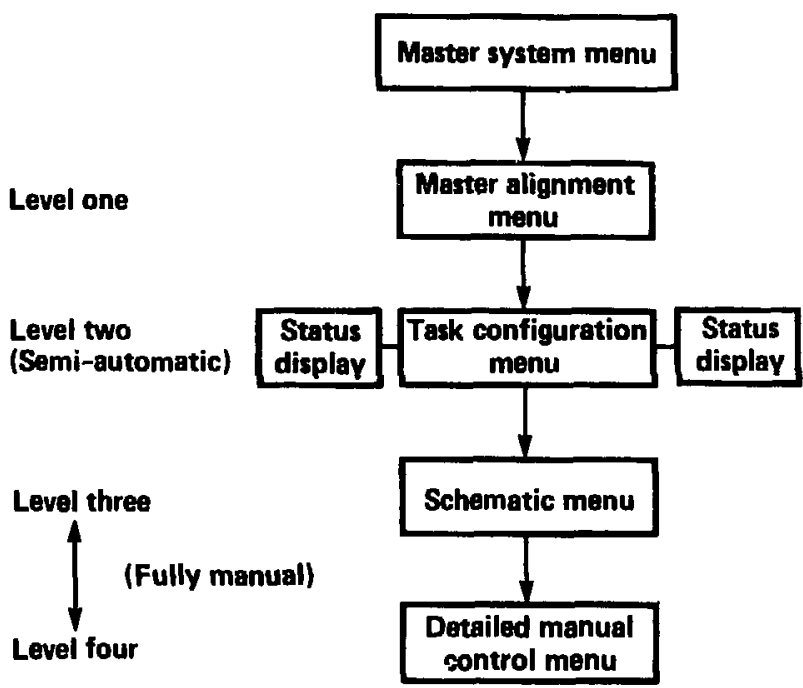

Fig. 4. The four-level control hierarchy in the CAP. The second level configures the alignment system for most tasks and initiates automatic alignment. The third and fourth levels provide for centralized manual controls. 
an alignment subsystem from several geographically ind functionally distinct areas of the laser facility. At level two, the operator can perform semi-automatic configuration functions, determine access to manual controls, and start automatic alignment sequences. For example, the taskconfiguration menu at level two offers commands for moving large groups of devices to predefined positions. The tasks to set up the system for common alignment activities and to request initiation of automatic alignment are defined in ASCII command files. The configuration commands follow a specific sequence, and the status of the system's configuration is checked to prevent system malfunction and possible laser damage.

Access to all devices is controlled at level two through the allocation system, which is maintained within each SMC for the device connected to it.
With the allocation system, multiple operator controls and the AAP may participate in nonconflicting tasks on the same alignment subsystem.

The menus at level three are schematic representations of portions of the laser (Fig. 5). Devices allocated for manual control are colored green on the schematic of the alignment subsystem and may be touch selected; nonallocated devices, colored grey, are not available to the operator for manual adjustments. Level-three menus allow the operator to select particular devices in the laser chain simply by touching the represented device. Two-stage device controls (such as XH (crosshair): IN/OUT) are directly available at level three. To move a cross hair in or out, the operator would touch the desired cross-hair representation in the beam-line schematic, the buttons labeled $\mathrm{XH}$ would light up, and the operator would touch either IN or OUT.

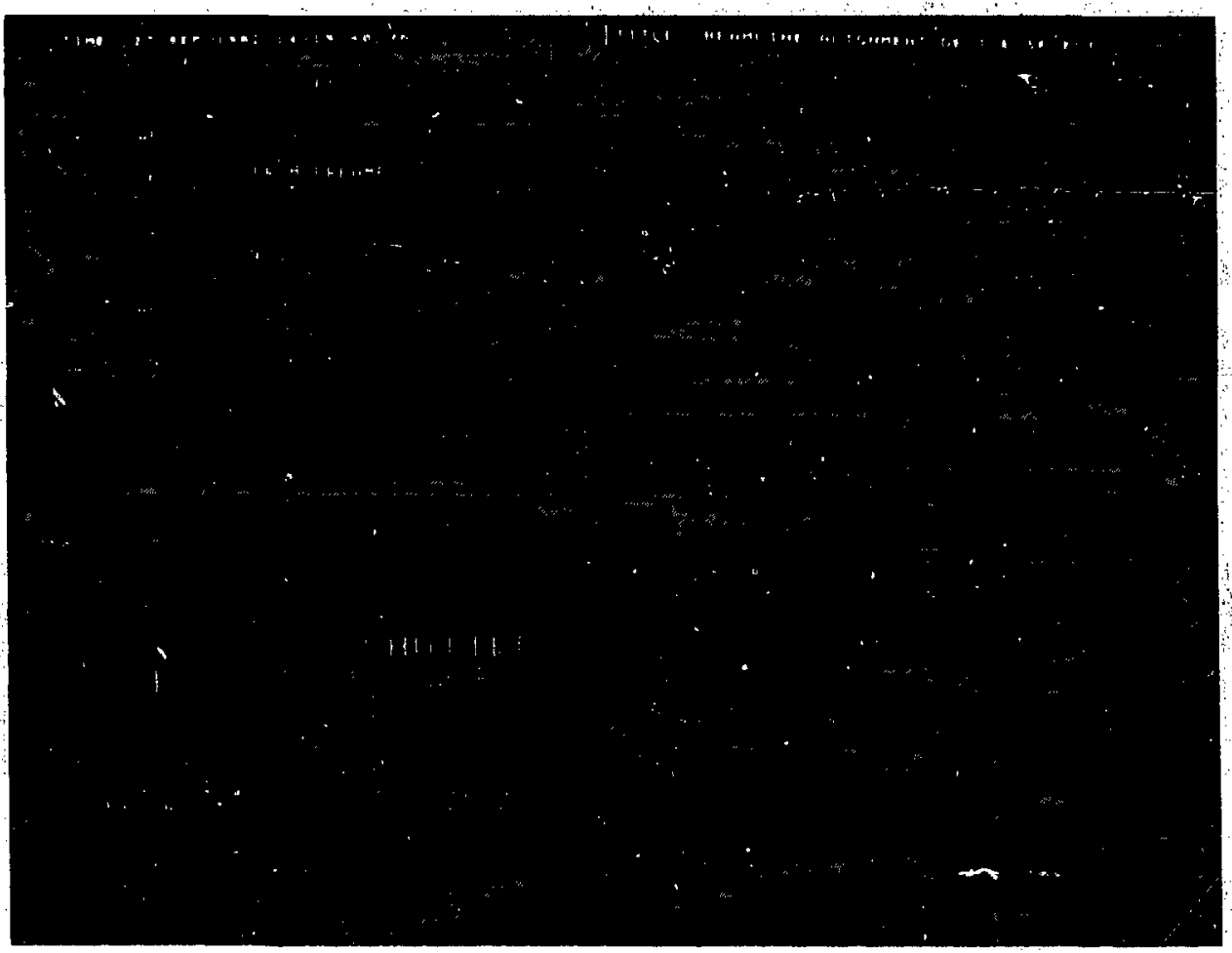

Fig. 5. The level-three, touch-sensitive menu screen. The screen provides schematic-oriented random access to many control elements from one menu page. 
Controls for more complex devices such as sensors and gimbals are available at level four. For example, to access the level-four, midchain-sensor manual controls, the operator would simply touch the box at the right of the screen labelled MS; the midchain-sensor, manual-controls touch menu would appear (Fig. 6).

Manual controls at level four allow the operator to perform specific alignment functions, such as continuous slewing of device motors. A separate joystick is used to enter simultaneously direction and rate in two axes. The joystick has finer resolution at the slow slewing rates to make small adjustments easier. Usually, the operator selects crosscoupling of motor coordinates to sensor coordinates such that the observed image motion tracks the joystick motion. Detailed status information is also displayed on the level-four graphics frame.

The ASP automatically displays the system status on the two side screens of the operator console as the control system adjusts alignment devices. Color-coded syinbols are used to summarize the current status of each aligrument device. Most conditions are displayed with continuous, solid colors-green to indicate a properly aligned component, or red to indicate an error condition. Blinking colors are used to indicate transient conditions, or to get operator attentionblinking white indicates a device is currently in motion. Text is used to augment the colored symbols for more specific device-status information. The color key is consistent throughout the alignment system.

The ASP automatically updates the status when the operator requests a particular frame through the CAP: when the graphics system displays the frame on the monitor, the ASP polls the appropriate SMCs to determine the current device status.

The independent nature of the ASP eliminates the need for any control program to maintain the status frames, which greatly simplifies the required

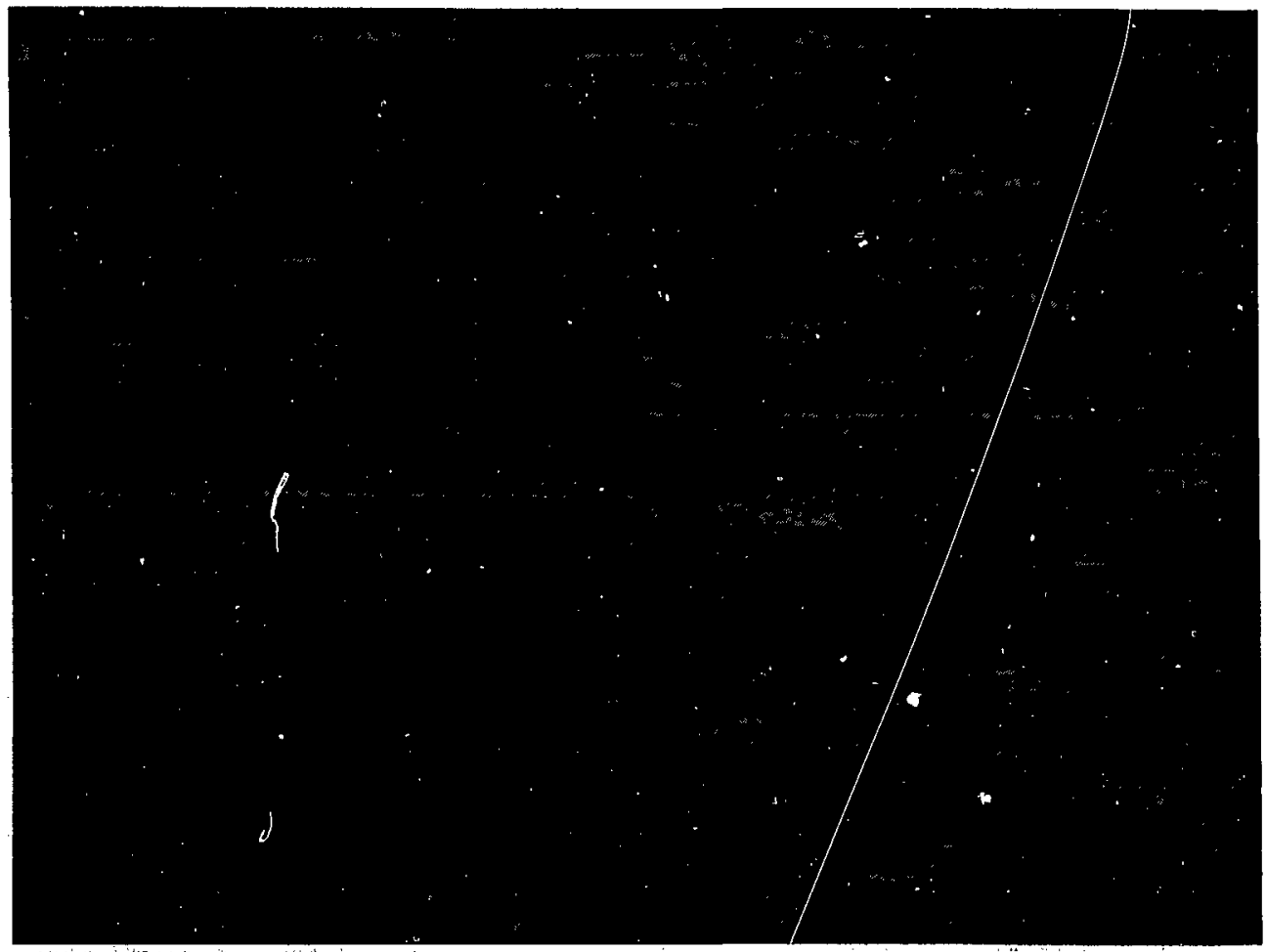

Fig. 6. Level-four, touch-sensitive menu screen. The midchain-sensor control frame provides detailed control and status of a single sensor. 
software for the control programs. Status displays are selected by operators as needed at any point in the menu tree. The ASP automatically determines which displays are in use and updates the status information in real time in parallel with any alignmènt procedures currently operating.

\section{Automatic alignment system}

The major component of the automatic alignment system is the AAP, which closes the pointing and centering loops of the laser beam by adjusting gimbaled mirrors. Pointing is the angular rotation of the beam about a single point, whereas centering is the parallel translation of the beam path. Once the beam is centered at the beginning of the optical chain, it is then pointed along the path to the next pair of mirrors without further affecting centering. Sensors in the system can separate pointing and centering information by obtaining far-field or near-field images of the laser beam. The AAP uses image-analysis techniques to locate reference features from each sensor's video picture. Corrections are then calculated to align the component. The procedure is repeated until errors are within tolerance.

The automatic alignment system requires additional, special instruments-a video-switching system, a video digitizer, and an array processor (which is necessary to accelerate the computationally intensive analysis). The video-switching system allows the operator to view any of the 43 alignment sensors in the facility, and is under software control by the AAP. Before the image can be processed, the video signal must be digitized to a $256 \times 256$ picture-element (pixel) format. The converted image is then processed by the array processor.

All video images are preprocessed to ensure consistent image quality. The images are subsampled down to a $128 \times 128$ pixel format to reduce both storage requirements and computation time. The resolution obtained is better than $1 \%$ of the sensor field of view.

Angular pointing information is seen as $X-Y$ translation on the TV monitor when the sensor is imaging the far field. The beam, when viewed by a sensor in pointing mode, is a small, focused, bright spot. The spot must be aligned coincident with a reticle on the sensor camera. The centroid of the image is calculated, giving the location of the focused spot. Pointing is most sensitive to alignment drift; therefore, these loops are closed more frequently than other alignment loops. Total loop iteration times of a few seconds, including the time necessary for motor positioning, have been achieved.

The reference points for centering the laser beam are given by the center of shadows cast from cross hairs temporarily inserted into the beam. To find the location of the center of a cross hair in an image, the image is first digitized without any cross hairs in the beam, then the cross hair is inserted and another image is digitized. Next, the image with the cross hair is subtracted from the image without the cross hair. Only the portion of the beam blocked by the cross hair remains. The first time this difference image is obtained (during the activation of the alignment loop) the location of the center of the cross hair in the image is identified by an operator and stored in the VAX along with the difference image as a reference template. Subsequent difference images, obtained during normal system operation, are correlated with the template to determine the current location of the cross-hair center. There is a unique template stored on disk files for each cross-hair image in the system. Cross-hair orientation is fixed, so only one correlation measure needs to be computed per image.

Figure 7, taken from a typical processing run, shows the three analysis steps observed by an operator at the console. Figure 7(a) is the cross hair as seen by the sensor camera. Figure $7(b)$, which shows the difference image, is correlated with the reference template by computing the twodimensional, fast Fourier transform on each image, and then by correlating in the frequency domain for maximum speed. Figure 7(c) shows the resulting correlation function, scaled to fit within the quantization levels of the video digitizer, and reordered spatially to restore symmetry about the correlation maximum. The location of the template in the image field is annotated by a cross superimposed on the original in Fig. 7(a).

For most sensors, comparison of two cross-hair images is used to determine corrections. One cross hair identifies the beam center, and the other is a reference for beam alignment. The above analysis is done for both cross hairs, one at a time. The centers of the two cross hairs are aligned to be coincident by driving the appropriate gimbal stepping motors. 

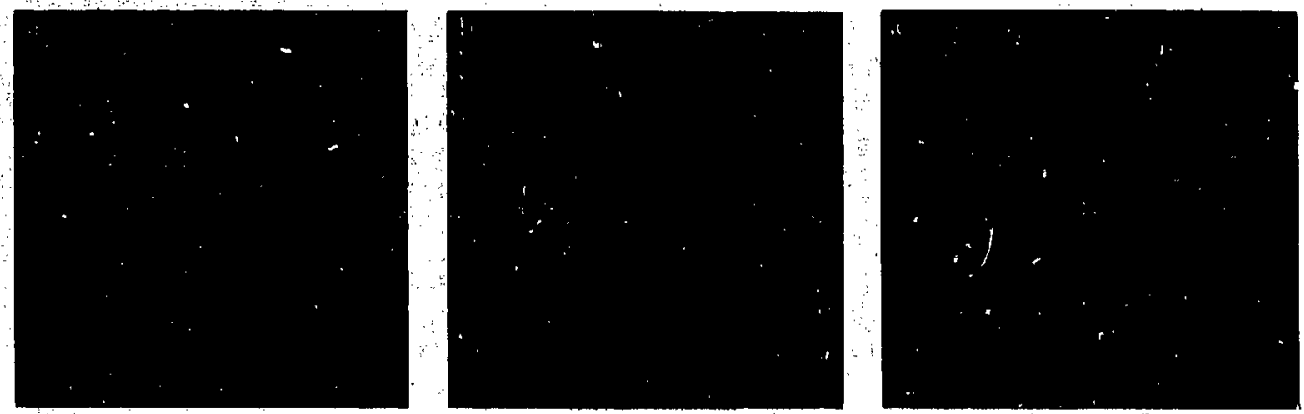

Flg. 7. Cross-hair analysis. (a) Sensor cross-hair image. (b) Difference image. (c) Correlation function.

The AAP operates by reading ASCII command and parameter lists, which are supplied in disk files. To perform a different task, a different command list is specified. The AAP continually searches through the command lists until told to stop by the console operator or by a supervisory program, the shot scheduler. The AAP is particularly adaptable to hardware changes because only the analysis methods are coded directly in software. The file structure also allows us to add new loops and make parameter sdjustments without recompiling the software.

The automatic alignment system is structured to process all ten beams in parallel. A high throughput is obtained because analysis can continue on some beams while cross hairs or other components are in motion on adjacent beams. We have been able to close pointing and centering loops to the output sensor in slightly over $\mathbf{3 0}$ minutes. Pointing only is achieved in a few minutes. Corrections in any loop require more time because device motions must be verified.

\section{Analysis reliability}

Comparison of the maximum value of the correlation function with a minimum reference value recorded under normal operating conditions is a common test used to verify that the template object has indeed been found in the image field. The value of the cross correlation is very dependent upon image brightness, and use of this test alone would result in excessive failure of many of the analyses in cases of low illumination. Extremely bright but arbitrary images also drive up the correlation maximum value even if the template object is not visible in the image field, thus incorrectly satisfying the minimum-value test. Testing the correlation against a minimum value in an experimental environment is not rigorous enough to ensure reliable analysis.

Another test to ensure accurate cross-hair reference detection is performed by comparing the histogram of high-valued correlation points to those obtained under normal operating conditions. Typically, only a small number of the total 16384 image points satisfy this criterion. Histograms of greater than two or three times the expected number of points indicate that the image quality is too poor to analyze with sufficient confidence. It has not been necessary tc check the actual shape or spatial distribution of the points, although such a step would be even more specific.

Application of the above test allows correlations to be accepted over a wide dynamic range with a high degree of confidence. The information present in the orthogonal arms causes the correlation function to peak sharply. The intersection determination is accurate unless only one arm can be seen (detected by the test outlined above). The same test also detects extreme over-illumination, or component damage effects where excessive numbers of image points have values near the correlation maximum value. 


\section{Conclusion}

The Nova alignment controls are easier to use and expand than were designs fielded for previous Livermore laser facilities. The modular design of the alignment controls allows both automatic and manual operations to be performed on completed areas of the facility while other areas are still being installed or are being maintained. Color graphics on the operator controls improve the alignment control and monitoring of a large optical system. Extensive automation simplifies the operation of the facility, improves reliability, and provides more consistent alignment. These factors combine to reduce the requirements on the laser operating staff, lower costs, and shorten alignment times.

\section{References}

1. P. J. Van Arsdall, F. W. Holloway, D. L. McGuigan, and R. T. Shelton, "Nova Laser Alignment Control System," Proceedings of SPIE-Intemational Society for Optical Engineering, Vol. 483, Optical Alignment II (Arlington, VA, 1984).

2. E. S. Bliss, R. G. Ozarski, D. W. Myers, J. B. Richards, C. D. Swift, R. D. Boyd, R. E. Hugenberger, L. G. Seppala, J. Parker, and E. H. Dryden, "Nova Alignment and Laser Diagnostic Systems," Engineering Design of the Nova Laser Facility for Inertial Confinement Fusion, Lawrence Livermore National Laboratory, Livermore, CA, CONF-811040 (1982), presented at the IEEE 9th Symp. on Engr. Problems of Fusion Research, Chicago, IL. 


\title{
Nova Beam Diagnostic System
}

\author{
D. Myers and R. Ozarski
}

\begin{abstract}
The Nova beam diagnostic system characterizes each of the ten Nova laser chains at key locations. Meașurements of beam energy, temporal pulse shape, spatial beam profiles, and system timing are made by a three-level computerbased control system. This article describes the architecture, operation, and capability of the system.
\end{abstract}

\section{Introduction}

The Nova beam diagnostic system allows us to achieve optimum irradiation of the target while avoiding optical damage to the laser components by fully characterizing each of the 10 Nova laser beams on each shot. ${ }^{1}$ Beam diagnostic data are used to reduce target diagnostic data and help laser operators adjust the laser for consistent and reliable performance. To characterize the laser beams, the diagnostic system performs the following tasks:

- Monitors the beam at key locations along each amplifier chain as it increases in energy to verify laser performance and to set each chain's output energy.

- Records temporal shapes of the input pulse from the oscillator, the amplifier chain output, and the output after frequency conversion to guard against damage and set the correct pulse shape for target experiments.

- Measures timing relationships between the laser pulse and other signals, such as Pockels cell voltages, to assist in the setup and troubleshooting of the laser.

- Verifies positioning of the arrays of potassium dihydrogen phosphate (KDP) frequency-conversion crystals to achieve maximum frequency conversion.

\section{Design and architecture of the system}

Nova's main laser bay is $200 \mathrm{ft}$ long, with laser chains on five separate levels and six diagnostic sensors per beam line. The design of the beam diagnostic system (Fig. 1) allows it to control the many widely distributed, inconveniently located sensors and to expand as additional diagnostics are required. ${ }^{2}$ This architecture consists of three separate control levels. The first is the sensor level, where the various diagnostic signals are acquired. The second level controls the first level sensors, does preprocessing of the beam diagnostic data and communicates with the third level. The third level integrates the first and second level functions into a cohesive data acquisition and analysis system.

\section{First level}

Beam diagnostic signals begin with the six basic elements of the first level:

- dc amplifiers

- Charge amplifiers

- Synchronous amplifiers

- Streak cameras

- Transient digitizers

- Positioning equipment

Energy measurements are made using absorbing glass calorimeters with dc amplifiers and silicon photodiodes interfaced to charge amplifiers. Synchronous amplifiers are used to tune KDP crystals with low-level, continuous-wave (CW) laser beams and to accomplish several systemmaintenance functions, such as focus, lens-transmission, and reflectivity measurements. Since these sensors must operate near the high-voltage, highcurrent discharges ( $22 \mathrm{kV}$ at $4 \mathrm{kA}$ ) of the 6000 flashlamp circuits of the laser amplifiers, their microvolt, picocoulomb, and microamp signals are amplified immediately and digitized to reduce EMI pickup and interference during transmission. A ROM-based communication-and-control module communicates with the second level over a serial data line, interpreting the incoming commands and providing the requested data or executing the control functions requested from the second level. 


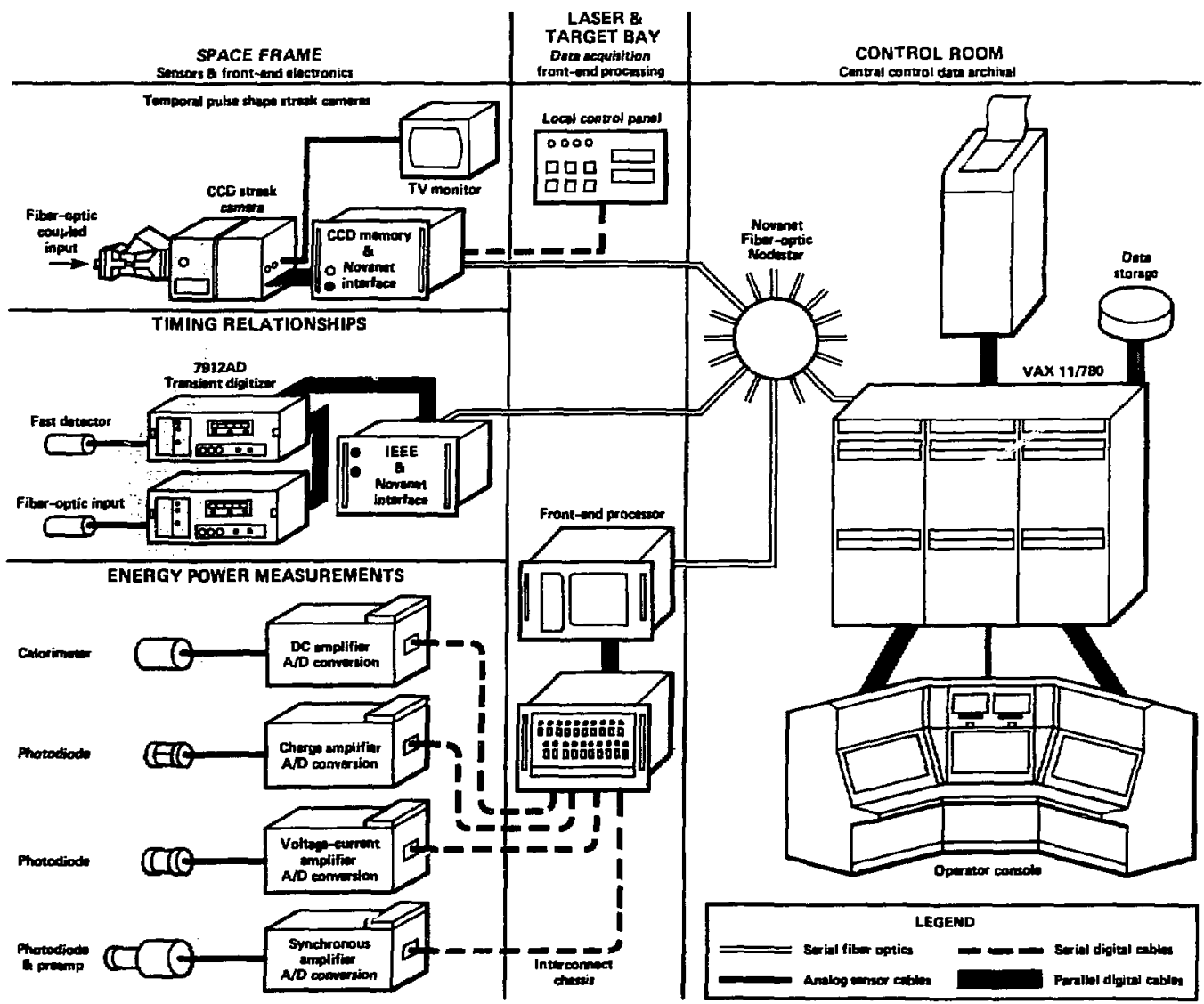

Fig. 1. Diagram of the overall beam diagnostic system. The first level is a network of diagnostic sensors. The higher levels of the system collect and process signals from the sensors, automatically reduce and archive data, provide user-friendly interfaces for operator control, and automatically sequence pre-shot diagnostic events. 
Identical hardware and packaging are used for all Nova signal amplifiers (Fig. 2). Each amplifier consists of communication-and-control module, an analog-to-digital conversion module, and a unique signal-conversion module that determines the function of the signal amplifier. Modular and common hardware elements are used to maximize the maintainability of over 200 widely scattered amplifiers. To ensure data integrity on a laser shot, the data acquisition system can apply a self-test signal to the input of each amplifier to verify correct amplifier operation.

LLNL-designed streak cameras with chargecoupled-device (CCD) camera readouts are used to measure the time history of the laser pulse as it propagates down the laser chains. The CCD camera is controlled directly from the top level with pulse information being stored in a local memory until it is required by the diagnostic system operator after the shot.

Tektronix 7912AD transient digitizers store timing waveshapes collected from various sensors scattered throughout Nova for laser setup and fault analysis. The second-level controller uses a standard IEEE-488 bus system for data transfer and control.

Many sensors have multiple functions that may be selected and used by positioning elements such as lenses, filters, and mirrors with mechanical actuators. Three types of actuators are used: dc stepping motors, ac motors, and solenoid-actuated pneumatic cylinders. Actuator control is provided by stepping-motor controllers (SMCs), which are part of the alignment system and are discussed further in that section of this issue.

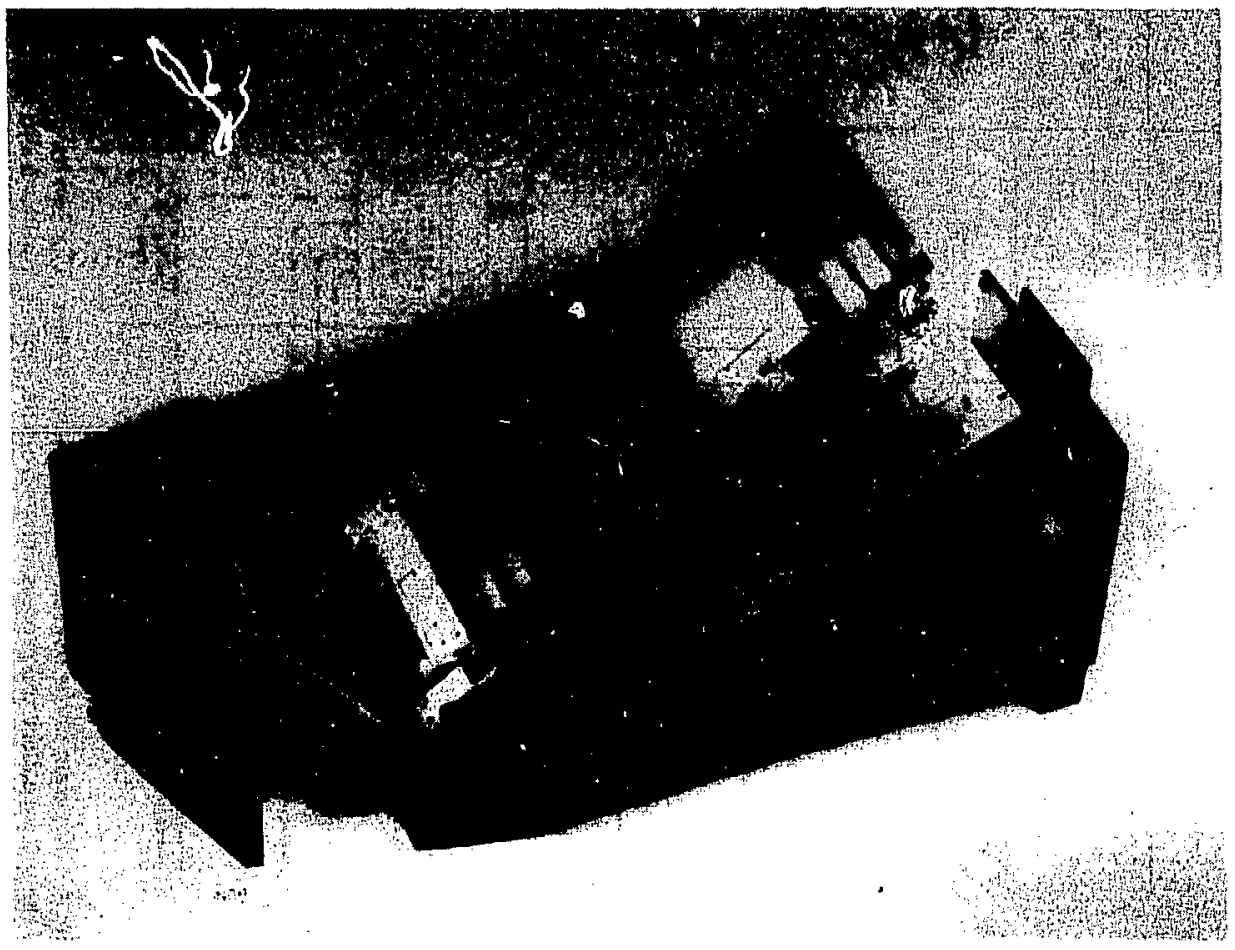

Fig. 2. A signal processor. All diagnostic-signal amplifiers are packaged identically. The top board is unique to the type of signal amplification required; the second provides analog-to-digital conversion; and the third provides standard communication and control. 
Second level

The second level provides centralized collection points for first-level sensors, instruments, or actuators. Through diagnostic energy processors (DEPs), remote device controllers (RDCs) and SMCs, the beam diagnostic system controls firstlevel devices, stores and reduces data, and communicates with the third level.

Eight DEPs are scattered throughout Nova to control various sensors. As a sensor controller, a DEP provides complete control over as many as 60 first-level sensors. It collects and reduces sensor data to the desired energy measurements, then transmits them to the third level, along with the raw data and control parameters, for archiving. The DEF shown in Fig. 3 uses an LSI-11/23 as its central processing unit (CPU), with additional interface boards to configure it for its beam diagnostic task. Each DEP has a set of sensor-independent interfaces (a control-panel interface, an amplifier-control interface, and a Novanet interface) as well as sensor-dependent interfaces (such as a calorimeterheater interface for DEPs that control dc amplifiers).

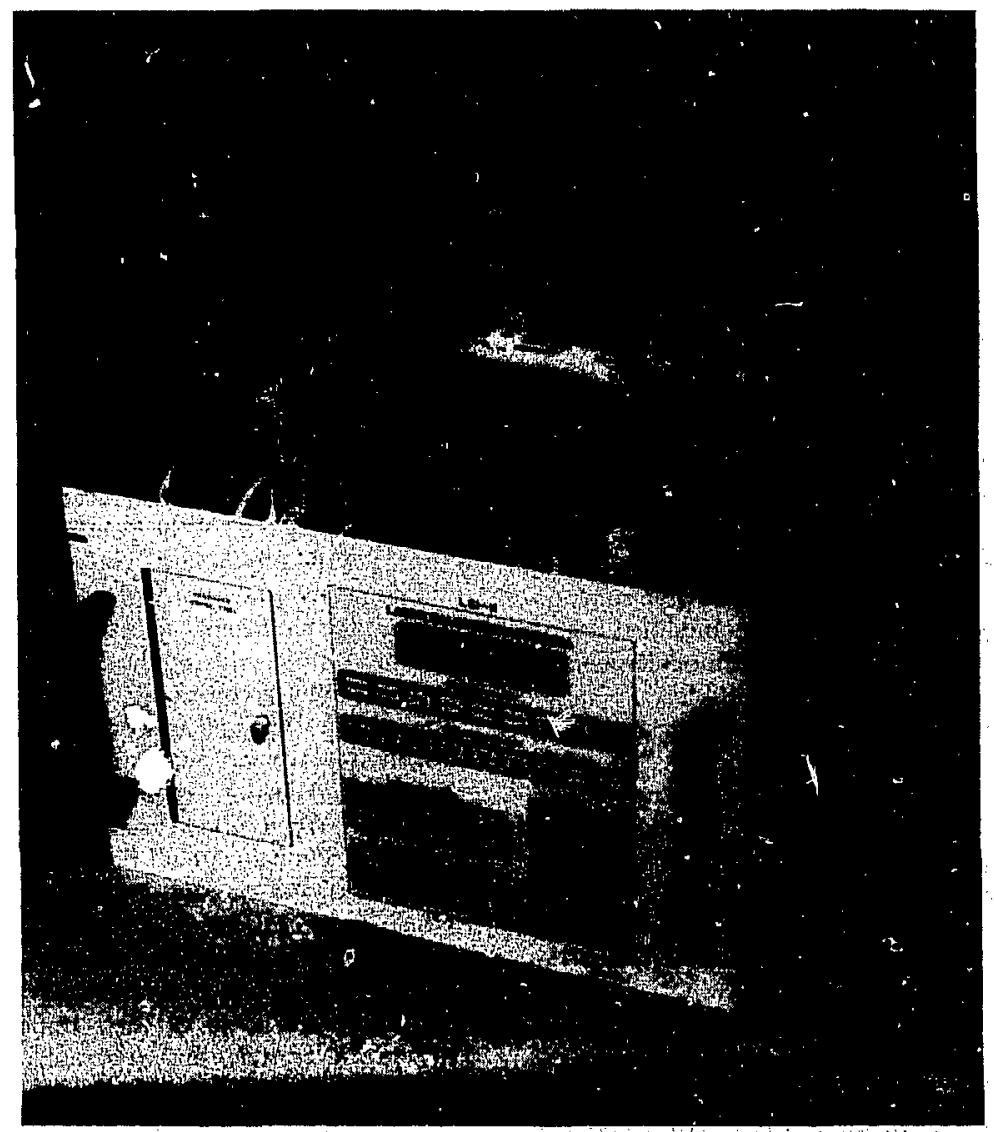

Fig. 3. A DEP and control panel. Although packaged identically to other DEPs, this DEP is programmed to perform a specific function. Using an overlay of labels unique to this control panel, an operator manipulates various sensors through switches on an underlying membrane switch panel, which includes a numeric-input keypad. Two vacuum-fluorescent displays (VFDs) display operator inputs and associated prompts. 
The control-panel interface allows universal con'rol of the different types of amplifiers, specialized functions, and unique outputs. On the panel, two vacuum-fluorescent displays (VFDs) display operator prompts, data, and messages. On a $9 \times 9$ matrix of membrane switches, covered by a removable plastic overlay, are printed the functions, tasks, and names of the individual sensors controlled by the DEP. Although all DEPs use common hardware, each DEP is pros, ramm?d to serve a specific function. The overlays , the control panel easy to understand, whir $r$ : rn allows the operators to control $\mathrm{m}_{\circ}$ uque sensors. In addition, the overlays are in movable, and thus interchangeable, so it is easy to add to or modify the diagnostic system.

The control-panel interface sends and receives data over a serial line with the same software protocoi as a printer or display terminal. This allows easy programming of the interface, and the serial line allows it to be removed from the DEP and used as a remote control. This is desirable when an operator controlling a sensor needs to observe the results of his actions on a control-panel display remote from the DEP.

The amplifier-control interface provides the control-and-data link between the DEP and its amplifiers. Control commands are sent to, and data received from, the amplifiers as serial command/ data words. A DEP may have as many as three of these interfaces, each of which enahles control of up to 20 different anplifiers. Because of the commonality designed into the system, all amplifiers use the same communication-and-control modules with the same protocol and are thus compatible.

Thr: system is made easier to maintair: by identifying each device with a number that can be interrogated by the DEP. This allows us to easily locate each device in the system and check its calibration or performance against previously determined values. Through periodic checks of first-level devices, we can detect changes or degradations in performance and correct them before shot data can be compromised.

The Novanet interface, described elsewhere in this issue, provides the communication link from the DEP to the third control level, where all parts of the diagnostic system are integrated. RDCs provide second-level control for instrumentation devices that contain their own local controls and memory and, therefore, do not require a DEP or SMC for control. These devices are controlled through an IEEE-488 instrument bus attached to a Novanet Q-bus device interface (QBDI).

\section{Third level}

At the third level, VAX-11/780 minicomputers provide overall control of the diagnostic system. The operator manipulates individual elements of the system from a touch-screen control console, where commands are processed and sent to the appropriate second-level controllers to obtain the desired data or initiate control actions. Periodically, the third level receives status updates on various processing functions from the second level, so that whenever a function or control is selected at the touch screen, the current status of the related set of sensors and functions is displayed on an adjoining color-graphics screen.

Three types of software are used at this level: manual remote control, integrated functional control, and automatic sequencing control.

Operators can manually set certain laser functions (such as sensor gains) by using a touch-screen control panel. The functions available at the DEPs are also available at the panels. Manual remote control also provides RDC-interfaced instrument control to the operator for devices such as the transient digitizers.

Integrated functional control permits the operator to control the syst. in from a functional rather than a device standpoint. For example, the operator selects a diagnostic sensor location from a touchscreen display map of the laser and selects the function he wishes to initiate. The software then routes the command to the particular DEP, SMC, RDC, or combination thereof to reach the appropriate sensor. Without this method, the operator would have to input a complicated device code to select the specific DEP and sensor and then execute the required function. Sophisticated and complicated data reduction required for orienting the frequency-conversion crystals is also done by iniegrated functional control. For this, a plot of signal level vs crystal position is obtained to determine what angle of the KDP crystal will achieve maximum conversion efficiency. The software takes measurements from the appropriate sensors, determines the ratio of their respective outputs, moves the crystals, and generates a plot for the operators.

Automatic sequence control sets in motion a series of events that must take place in preparation for a laser shot. After the type of shot and some shot conditions (such as the pulse length to be used and the laser amplifiers to be fired) are selected, the auto-sequence software uses these data to define the type of sequence to be executed. First, the software 
determines and sets the orientation of the frequency-conversion arrays for maximum conversion efficiency. Next, it configures diagnostic sensors for the wavelength to be used on the target and sets them to gains appropriate for the expected: energy levels. Then, it exercises the internal test procedures to ensure accuracy and readiness for a shot. A few minutes before shot time, the diagncstic system starts the acquisition of data. After the shot, it reduces and sends shot data to the video displays on the control console, prints a hard copy, and archives the data on disk.

Archived data can be retrieved from storage by keying in the shot number and requesting data recall at the touch panel. We can then compare archived data from previous and subsequent shots to detect changes in laser performance and to determine how laser performance affects target performanice.

\section{Conclusion}

The design of the Nova beam diagnostic system allows us to accurately characterize the Nova laser beams, providing data that allows the system opera: ors to achieve optimum irradiation of laser fusion targets while avoiding optical damage to the laser. Also, the system provides laser output data for analyzing target experiment results, determining laser performance, and diagnosing laser system problems. The system's threalevel architecture permits expansion, flexibility, and easy maintenance.

\section{References}

1. D. W. Myers, R. G. Ozarski, !. R. Richards, and J. L. Wilkerson, "Nova Laser Beam Diagnostic Data Acquisition System," Technical Digest of the Conference on Lasers and Electro-Optics (Anaheim, CA, 1984).

2. E. S. Bliss, R. G. Ozarski, D. W. Myers, J. B. Richards, C. D. Swift, R. D. Boyd, R. E. Hugenberger, I.. G. Seppala, J. Parker, and E. H. Dryden, "Nova Alignment and Laser Diagnostic Systems," Engineering Design of the Nova Laser Facility for Inertial Confinement Fusion, Lawrence I: rermore National Laboratory, Livermore, CA, CONF-811040 (1982), presented at the IEEE 9th Symp. on Engr. Problems of Fusion Research, Chicago, II.

3. R. G. Ozarski, "Beam Diagnostics on a Multi-Wavelength Fusion Laser," Laser Focus 17(12), 51 (1981). 


\title{
Nova Target-Diagnostics Control System
}

\author{
J. Severyn
}

\begin{abstract}
The Nova target-diagnostics control system has the task of recording signals from diagnostics instruments that have a wide range of bandwidths and signal levels. Our system, which includes acquisition and control functions using CAMAC hardware, is menu driven and experiment oriented.
\end{abstract}

\section{Introduction}

The Nova target-diagliostics system must record a wide variety of signals from many diagnostics instruments ${ }^{1}$ surrounding the laser fusion target. The intense temperatures and pressures created at the target by focused laser beams produce a fusion reaction that emits subatomic particles and energy. Instruments to diagnose this reaction include $x$-ray, light, and particle detectors that range from $C C D$ streak cameras with 10-ps resolution, pinotodiodes and $x$-ray diodes with 100 -ps response, pliotomultipliers and silicon photodiodes with 1- to 2-ns response, to light and particle calorimeters with response times measured in full seconds. This wide range of bandwidths and variation in signal levels means that close attention must be given to diagnostic isolation and grounding to prevent cross talk and signal degradation.

The system must also be flexible to accommodate different target designs and changing diagnostic scenarios. Because the fusion target and most of the diagnostic detectors require a high-vacuum environment to operate properly, we have included an integrated vacuum control system to aid in the setup and reliable operation of these diagnostirs.

\section{Hardware architecture}

The Nova target diagnostics data acquisition system is organized around LSI-11 frontend processors (FEPs) and based on standard CAMAC hardware and protocols. Geographically distributed LSI-11s acquire data and perform control functions in the Nova laser target room. All diagnostics are isolated from the target chamber, the space frame, and grounds within the building.
Inputs and outputs for all diagnostic areas are isolated by the use of fiber optics. The ac lines are the sole exception, and they are isolated by lowcapacitance power transformers and a single-point ground to eliminate ground-loop current effects.

All digital communication and trigger signals to the diagnostic recording instruments and their associated FEPs are transmitted via fiber optics (Fig. 1). This allows the FEPs to be located near the signa!-digitizing equipment, thus minimizing the length of analog signal cables while maintaining strict isolation from the high-energy capacitor banks used to fire the laser flashlamps.

In the Nova building the diagnostic instrumen's are mounted on the target chamber primarily along a vertical ring that is aligned on a north-south ixis. Accordingly, we have chosen two locations for placement of digitizers: a room to the north (the diagnostics loft) and an area to the south in the switchyard. Single-shot transient digitizing equipment is not installed in the target room proper because of signal distortion caused by electromagnetic pulse (EMP) effects when the fusion target is imploded.

Diagnostic detectors are typically mounted on line-of-sight tubes bolted to the main target chamber. These detectors are usually either arrays of photomultiplier tubes, $x$-ray diodes, or photodiodes with various filters to allow the detector to respond to preset areas of the $x$-ray or optical spectra of interest to the target diagnostician (Fig. 2). These detectors are electrically isolated from the chamber, and the signal is cabled through isolated signal trays to the nearer digitizing room (either the diagnostic loft or the laser switchyard). We use air dielectric coaxial cable for wide-bandwidth signals, to minimize degradation while the signal is still in an analog format. 


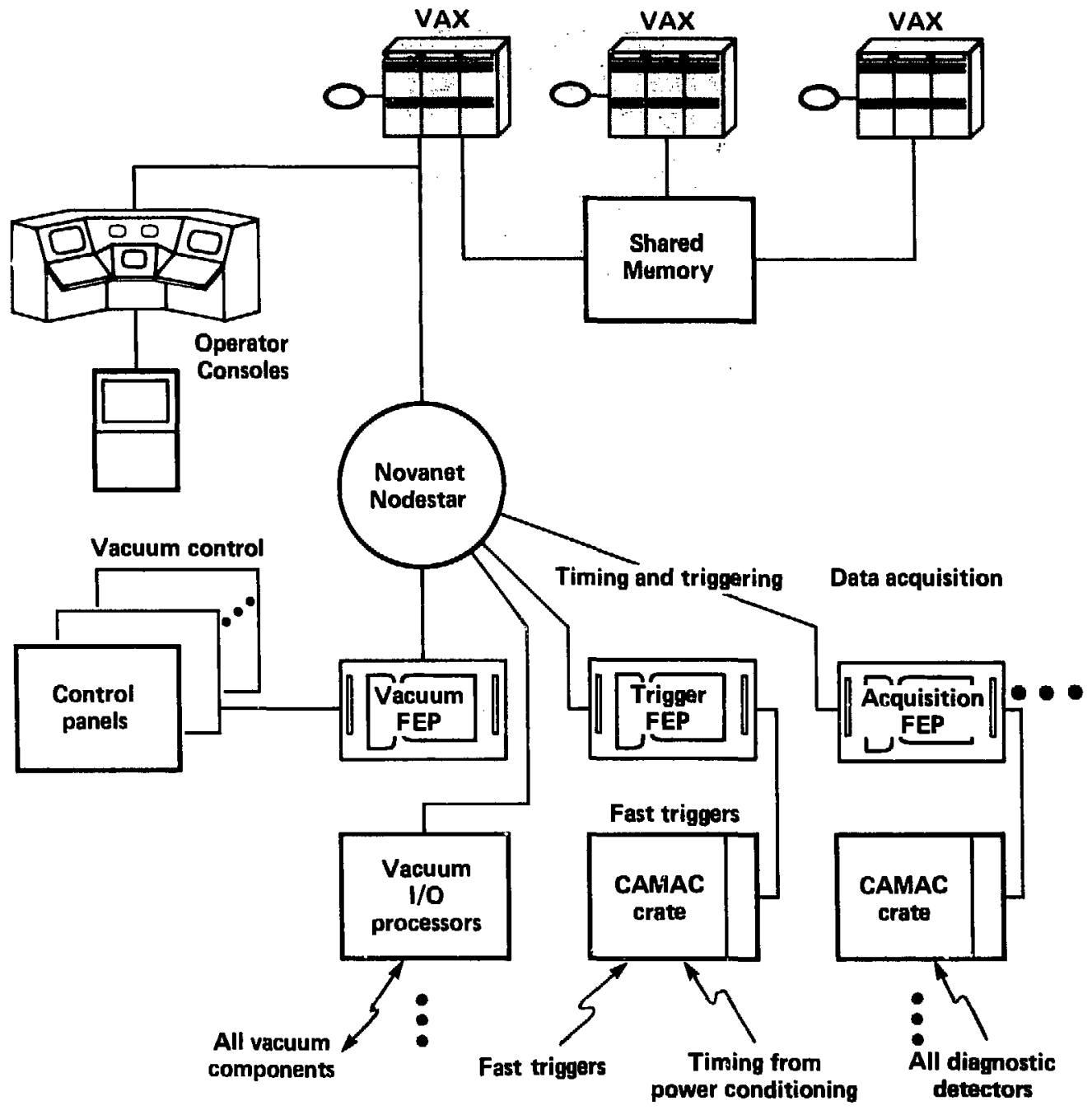

Fig. 1. Nova target-diagnostics control-system architecture. Signals between VAXs, FEPs and diagnostic recording instruments are transmitted through fiber-optic cables connected to the Novanet Nodestar distribution center. Fiber-optic links are immune to noise spikes inherent in analog signal cables and permit the FEPs to be located near signal-digitizing equipment. 


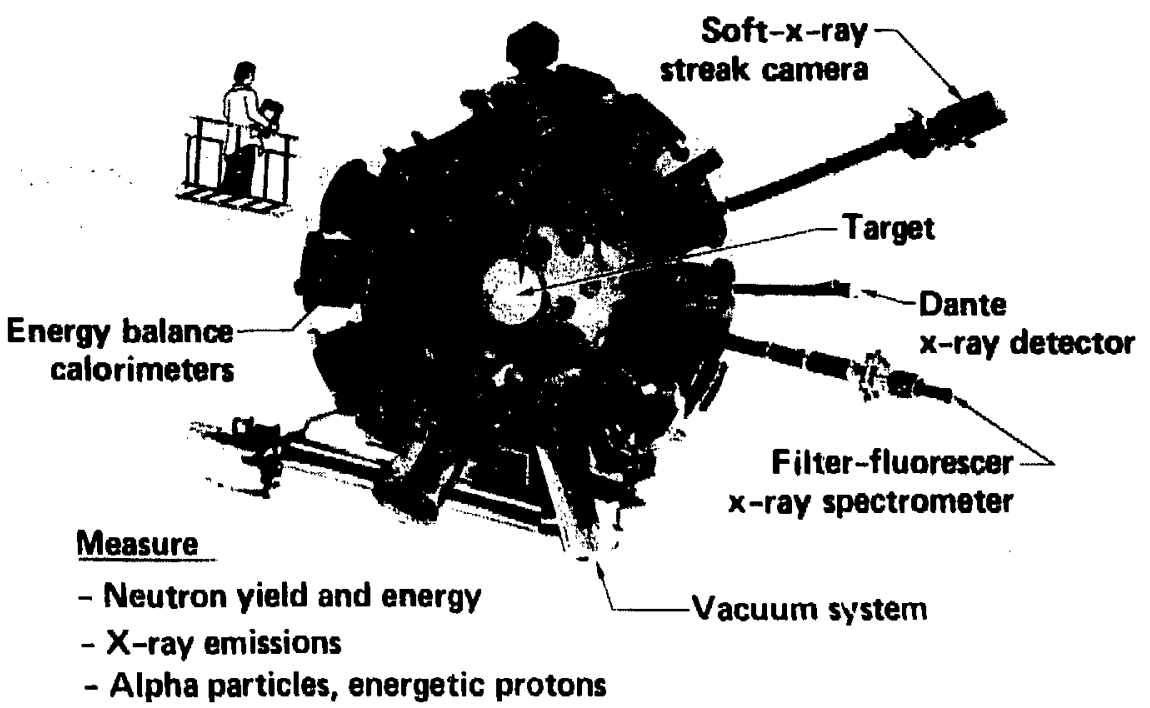

Fig. 2. Configuration of diagnostic detectors surrounding the target vacuum chamber. The detectors consist of arrays of photomultiplier tubes, $x$-ray diodes, or photodiodes equipped with filters that respond to the specific regions of the $x$-ray or optical spectra that the target diagnostician has selected for analysis.

In both the diagnostic loft and the switchyard, the signals are routed to the appropriate digitizer interfaced to CAMAC crates attached to the FEPs. Signals up to $1-\mathrm{GHz}$ bandwidth are digitized by Tektronix R7912 transient digitizers. Various CAMAC charge-integrating digitizers serve medium-bandwidth photodiodes, while multiplexed analog-to-digital converters handle lowbandwidth signals such as those from calorimeters. Some diagnostics require that optical or $x$-ray streak cameras be placed directly on the target chamber. In these cases, a film pack holds the recording medium, and the control system merely cycles the camera shutter mechanism and verifies that the camera time base has functioned properly during dry-run sequences and actual target shots.

Separate LSI-11 FEPs are provided for certain diagnostics (e.g., copper and lead neutron-activation counting systems and radiation-chemistry diagnostics) where a dedicated microprocessor allows convenient software partitioning for specialized tasks with dedicated hardware that change infrequently.

A target-diagnostics triggering and timing system is included to provide isolated electrical triggers to all diagnostics. It also provides timing fiducials that are included with many diagnostic signals to allow cross timing between channels within
50 ps. This system receives-from the Master Oscillator Room (MOR) - a fraction of the laser pulse that serves as the seed generator for the entire system. This fraction of the MOR pulse is transmitte' via fiber optics to the diagnostics loft where the trigger-system converts the optical pulse to an electrical equivalent, compensates for amplitude variations, monitors and records timing drifts, and distributes the triggers to four subsystems (Fig. 3). The trigger system serves four primary subsystems:

(1) First the instrument's triggers fire the time bases of digitizers, oscilloscopes, and standard recording equipment with approximately 300 ps of jitter.

(2) A separate streak-camera triggering system satisfies the 50-ps jitter requirements mandated by ultra-fast streak cameras.

(3) Third, the instrument fiducials are generated for inclusion with the digitizer and oscilloscope data to allow precise cross timing of diagnostic data.

(4) Finally, a signal simulator is included to allow accurate timing of diagnostics without the use of the main laser. This is accomplished by precisely generating and timing a simulated diagnostic signal and applying it to the same signal cables that will be used on the actual target shot. 


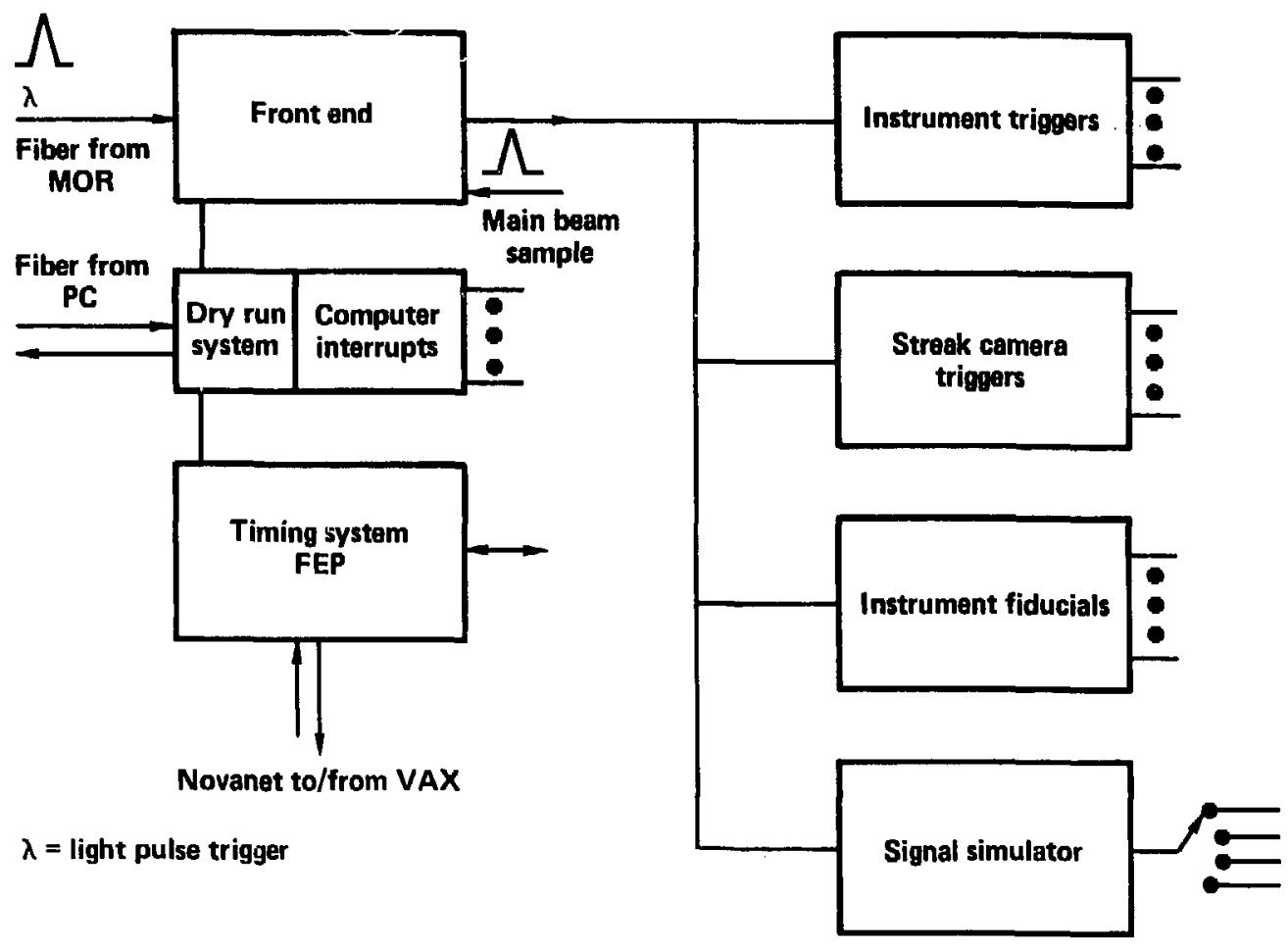

Fig. 3. Block diagram of target diagnostics triggering and timing system. The Master Oscillator Room (MOR) supplies a fraction of the laser pulse to the triggering system's front end, where the pulse is converted to an electrical signal and is distributed to four subsystems.

During the setup sequence and on the actual target shot, trigger delays and critical pulse amplitudes are monitored and logged both to alert the operator to improper conditions and to record actual shot conditions for later data analysis.

All LSI-11 FEPs are connected to one of the three Digital Equipment Corporation (DEC) VAX computers through the Novanet fiber-optic localarea network (LNN). Novanet's 10 megabit/s line rate permits rapid control of the FEPs for setup before each shot, and quick data collection from the array of digitizers after each shot. Data from a typical mix of diagnostics are collected by the VAX and stored on disk in about two minutes.

\section{System software design}

Our experience on previous lasers has shown that the target diagnostics system must remain flex- ible to allow easy changes in the hardware configuration. This requirement is most easily met by a software design that is "configuration file" driven: i.e., a minimum of configuration information is hard-coded into the system; instead, information is maintained on files that are read at setup time. The operator selects the configuration file most nearly describing the next shot, and modifies it to meet exact shot requirements, using the color-graphic touch panel. The configuration consists of a basic "shell" of hardware necessary for proper FEP and timing-system operation. The configuration file also defines groups of hardware that are physically associated with a particular diagnostic experiment. By choosing the diagnostics that are on-line for a particular shot, the operator also automatically selects the necessary set of hardware.

The basic software architecture is shown in Fig. 4. The VAX control code is implemented as a set of processes communicating through a common 
shared memory. The task is partitioned into a set of logical operations (processes) that all use a shared memory, which is physically resident in the MA780 memory (common to all three VAX computers used to operate the Nova laser). Because this memory is also common to all three VAX computers, access to other subsystems is also possible. For instance, most preshot timing information from the powerconditioning system is passed through common memory. The target system passes status information back to the power-conditioning system to complete a software interlock during the firing sequence.

The software interlocks during both the firing sequence and postshot data acquisition include the status of the FEPs. For most of the preshot setup sequence each FEP acts as a slave to the VAX, performing commands and passing back status upon request. However, for the last $15 \mathrm{~s}$ before the target shot, FEPs independently har,dle the final interrupt triggers and carnera-shutter sequences.
After the shot, they again become slaves to the VAX for collecting the shot data. This technique includes all physical address locations in the Novanet commands to the FEPs and, therefore, it is not necessary for diagnostic configuration information to actually reside in the FEPs.

An FEP accepts commands from the VAX, strips off the address information imbedded in the command, and then performs the required operation. (Typical commands include TURN ON HIGH VOLTAGE or READ 7912 DATA.) The FEP then performs the sequence of CAMAC operations necessary to do the operation. In some instances this can take up to several seconds of low-level bit manipulations on the hardware. It is during this interval that the frontend processor is used to offload these time-consuming operations from the VAX. After the command is completed, the FEP sends a single message back to the VAX that tells the status and-if the command entailed data acquisition-relays the data acquired.

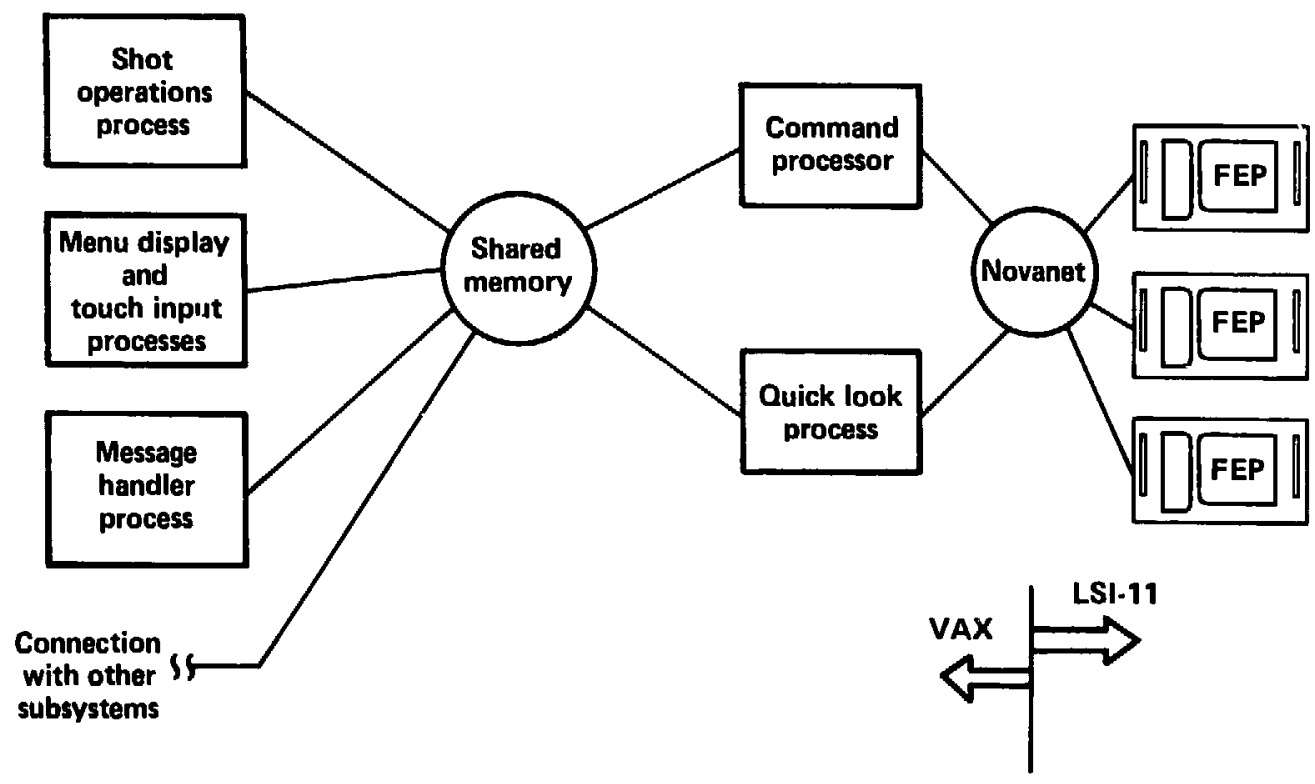

Fig. 4. Block diagram of the target diagnostics software architecture. The VAX control commands are transmitted to FEPs through memory shared by all three VAXs. Such memory sharing permits the partitioning of tasks into sets of logical operations and permits access to other subsystems. 


\section{Diagnostics vacuum control system}

The fusion target and most of the detectors used to diagnose the target are mounted in or on the Nova target chamber. The target chamber is a sphere $2.2 \mathrm{~m}$ in radius. The beam-focusing optics are mounted on the east-west hemispheres, and the diagnostic detectors are mounted on the surface of the chamber and on a 1-m-wide vertical ring that joins the two hemispheres (Fig. 2). The vacuumcontrol system architecture is similar to our other FEP-bused systems except in its emphasis on realtime control and fast response, which are necessary to protect expensive diagnostic equipment. As shown in Fig. 5, it consists of a main FEP attached to an array of local control panels and several (as many as six) input/output processors (IOPs). The typical diagnostic instrument and the main chamber are connected to the vacuum control system through th: IOP units. The IOPs supply 24-V-de to operate the valve solenoids; sense the valve state switches to determine valve position; and read, linearize, and scale the various vacuum gauges. The IOP also controls an array of compressors that operate cryogenic cold heads on both the main vacuum vessel and on each diagnostic. These cold heads cryogenically pump the main chamber and the diagnostics, maintaining pressures in the MTorr range. The compressors are connected in parallel in a closed loop and pump gaseous helium. The vacuum-control system activates compressors as needed to maintain sufficient line pressure to operate the cold heads.

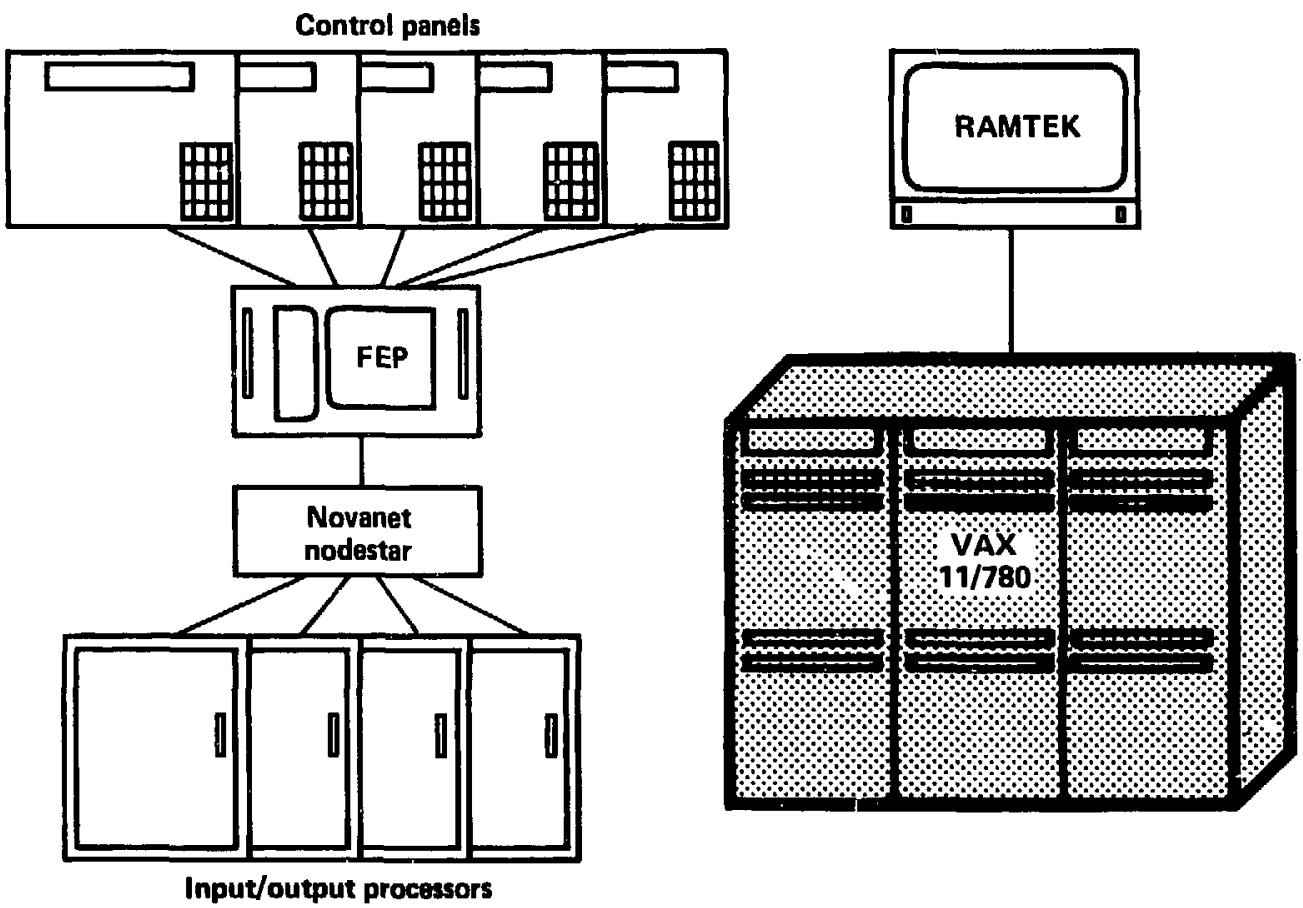

Fig. 5. Vacuum system controls hardware architecture. The configuration is similar to that of other FEPbased systems in that it consists of a main FEP coupled to an array of control panels and up to six input/ output processors (IOPs). The diagnostic instruments and the main chamber are connected to the vacuumcontrol system through the IOP. The control panels are positioned for convenient use near individual diagnostics in the target room. The main difference between the vacuum system and other FEP-based systems is in its emphasis on real-time control and fast response, which are necessary to protect expensive diagnostic equipment. 
Configuration and interlock information is downloaded into the main FEP before startup. The diagnostic control panels are attached to this FEP and are positioned where convenient for operator use in the target room near the individual diagnostics. These local control panels are identical units except for a plastic overlay that customizes the panel with the vacuum map of the diagnostic. Communication between the panels and the main FEP is via fiber optics (RS-232 equivalent signals are generated in an optical format), while communication between the FEP and the IOPs is via Novanet.

Once the main chamber and the individual diagnostics are operating, status and operator commands are entered through the console graphic touch panels used throughout the Nova control system. We monitor and control the global vacuum-system with the touch consoles in the diagnostics loft and the Nova control room.

\section{Conclusion}

The Nova target diagnostics system controls and collects data from a variety of instruments.
Excluding image data, approximately 1 megabyte of data are collected during the $\mathbf{2}$ min immediately following a target shot. Raw data are plotted for on-line, quick-look analysis. Further extensive analysis is done on a separate VAX reserved exclusively for such purposes. File transfer to the analysis VAX is accomplished by a one-way version of Novanet to protect classified data bases resident in the analysis VAX. The target diagnostic system is integrated into the Nova laser control system so that the control menus are organized consistently. This allows efficient control of complex diagnostic instruments.

\section{Reference}

1. H. G. Ahlstrom, "Diagnostics of Experiments on Laser Fusion Targets at LLNL," Physics of Laser Fusion, Vol. II, Lawrence Livermore National Laboratory, Livermore, CA, UCRL53106 (1982). 


\title{
Nova Shot Scheduler
}

\author{
T. De Groot
}

This article describes the design and operation of the Nova shot scheduler, which is the software system that operates the Nova laser facility. It coordinates the functions of the four Nova subsystems, the descriptions of which are presented elsewhere in this issue. The scheduler provides a means of executing control-system tasks in a desired order, or simultaneously, as the case may be. Operators interact with the shot scheduler through menu-driven consoles with high-resolution, color-graphics displays. Data files provide all task descriptions and task-ordering information needed to schedule the desired control tasks. The scheduler also automatically logs significant events for archiving.

\section{Introduction}

The Nova shot scheduler facilitates the scheduling of a complex array of control-system tasks that need to be done by the Nova subsystems. The scheduler ensures that the tasks are done at the correct times and in the correct sequence to properly operate the laser facility. For example, tasks that involve aligning laser components are sequenced through the scheduler and then performed by the alignment subsystem.

Laser operations personnel interact with the scheduler using central control consoles, which are described elsewhere in this issue. Hierarchical menu-driven displays allow an operator to select status options and the level of detail required for proper control. Status of lower-level components is summarized in the upper-level menus. Displaying only the desired level of detail reduces screen clutter and simplifies operation.

In a complex, ever-changing experimental environment such as Nova, new experiments impose new control-system requirements. The scheduler is flexible enough to accommodate such requirements. By the same token, the scheduler will help us to gain improved task-dependency information as we increase our laser operating experience.

\section{Scheduler design}

The scheduler displays the tasks to be performed as a data-flow diagram ${ }^{1}$ or bubble chart, so-called because the tasks are represented by circles (bubbles). The arrows in the diagram indicate task dependencies. If execution of a task is dependent on the completion of another, an arrow is drawn from the completion task to the dependent task. The arrow represents an "output dependency" for the completed task and an "input dependency" for the dependent task. A task is immediately started when all its input dependencies are satisfied. This data-flow design provides any desired type of task ordering and promotes task parallelism. Executing tasks in parallel reduces total task-execution time, thereby increasing controlsystem throughput.

The label in each bubble indicates a specific task to be performed. This may be either a description of a function that performs the task directly or a submerged data-flow diagram of subtasks, the sum of which performs the indicated task. In this manner, large data-flow diagrams are broken down into smaller, more understandable diagrams.

Determining the relationship of a given lask $A$ to any other task $B$ is simplified by arranging the tasks into a tree structure. In a task tree, each task is associated with a finite number of disjoint tree structures, called subtrees. Each of the subtrees corresponds to a subtask or "descendiant" in the data-flow diagram for the "ancestor" task. Task A is a direct ancestor of task B if and only if task B is part of task A. Figure 1 shows a set of tasks arranged in hierarchical data-flow diagrams and in a tree structure. In the task tree, tasks B, C, and D are direct decendants of $A$, since they are directly below $A$, their direct ancestor. In task $A^{\prime}$ s data-flow diagram, one can see that task $A$ is composed of tasks B, C, and D. Similarly, tasks E, F, G, H, and I 
are direct descendants of task $\mathrm{C}$, their direct ancestor. Task C's data-flow diagram shows that task $C$ is composed of tasks E, F, G, H, and I. Task $A$ is the ancestor of all the other tasks, since all are below task $A$ in the tree.

A task can be either automated or manual. The control system performs an automated task as soon as it is cleared of input dependencies. When the task is completed, all its output dependencies are satisfied. Any new tasks that are then executable are i: umediately started. The scheduler communicates a manual task to the operator via the console. The operator performs the task and then notifies the scheduler via the console that the task is complete. The scheduler then automatically satisfies the output dependencies for the task and continues the scheduling sequence. In a way, the scheduler is an automated checklist. Instead of operators being required in list and check off tasks on a piece of paper, the scheduler does these functions automatically, including logging the start and completion times for each task.

\section{Scheduler man-machine interface}

The operator controls all tasks by interacting with the scheduler through the central control console, where he selects options (from a control frame and menu displayed on the center screen) to perform desired control functions. Figure 2 shows a typical control-frame display, which consists of a data-flow diagram, or graph, above contactsensitive control "buttons."

The status of a task is indicated by the color of the corresponding bubble in the frame. Table 1 shows the possible types of task status. The status of a task implemented by subtasks in a submerged data-flow diagram is a status summary of those subtasks. In this way, the operator is aware of the status of many tasks without watching them individually. However, if there is a "holding" indication on the bubble of an anr.estor task, the operator can trace through the task's tree until the problem is found.
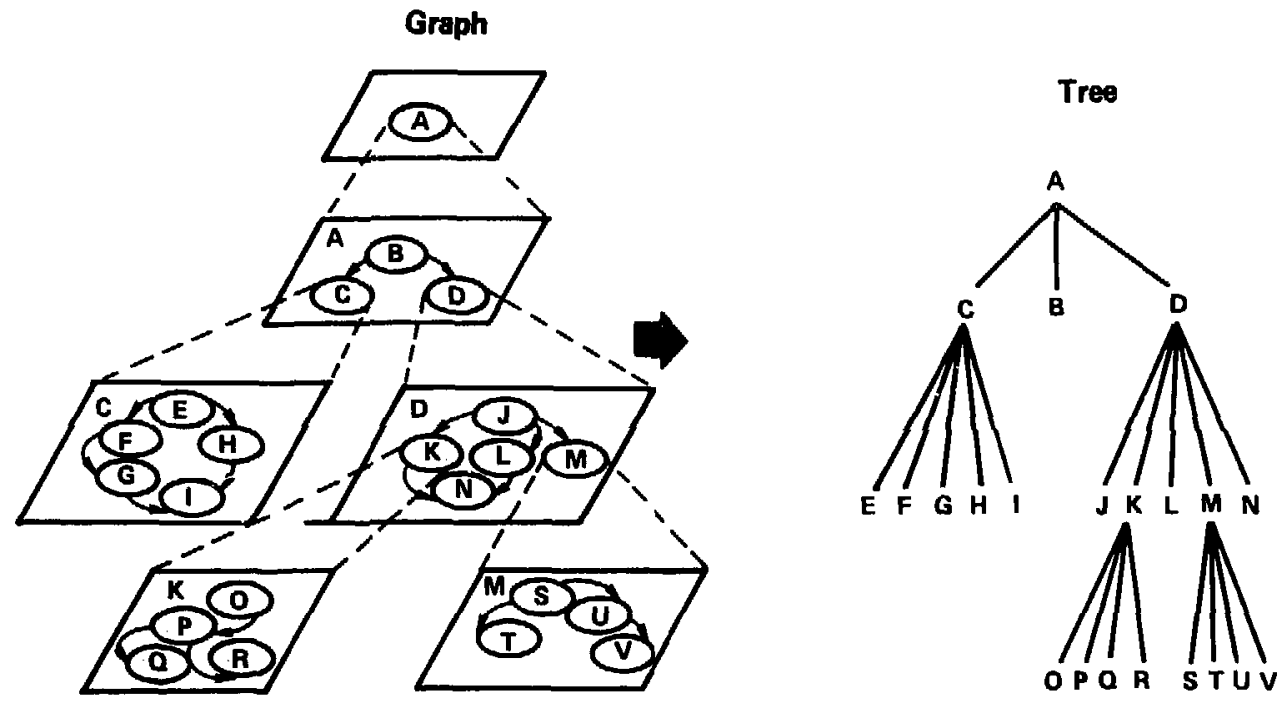

Fig. 1. Hierarchical data-flow diagrams. The data-flow diagrams on the left are mapped to a tree structure on the right. The tree structure shows the relationship between any of the tasks. For example, the data-flow diagram shows that task $B$ is part of $A$. The tree structure shows that task B is a descendant of task $A$. 


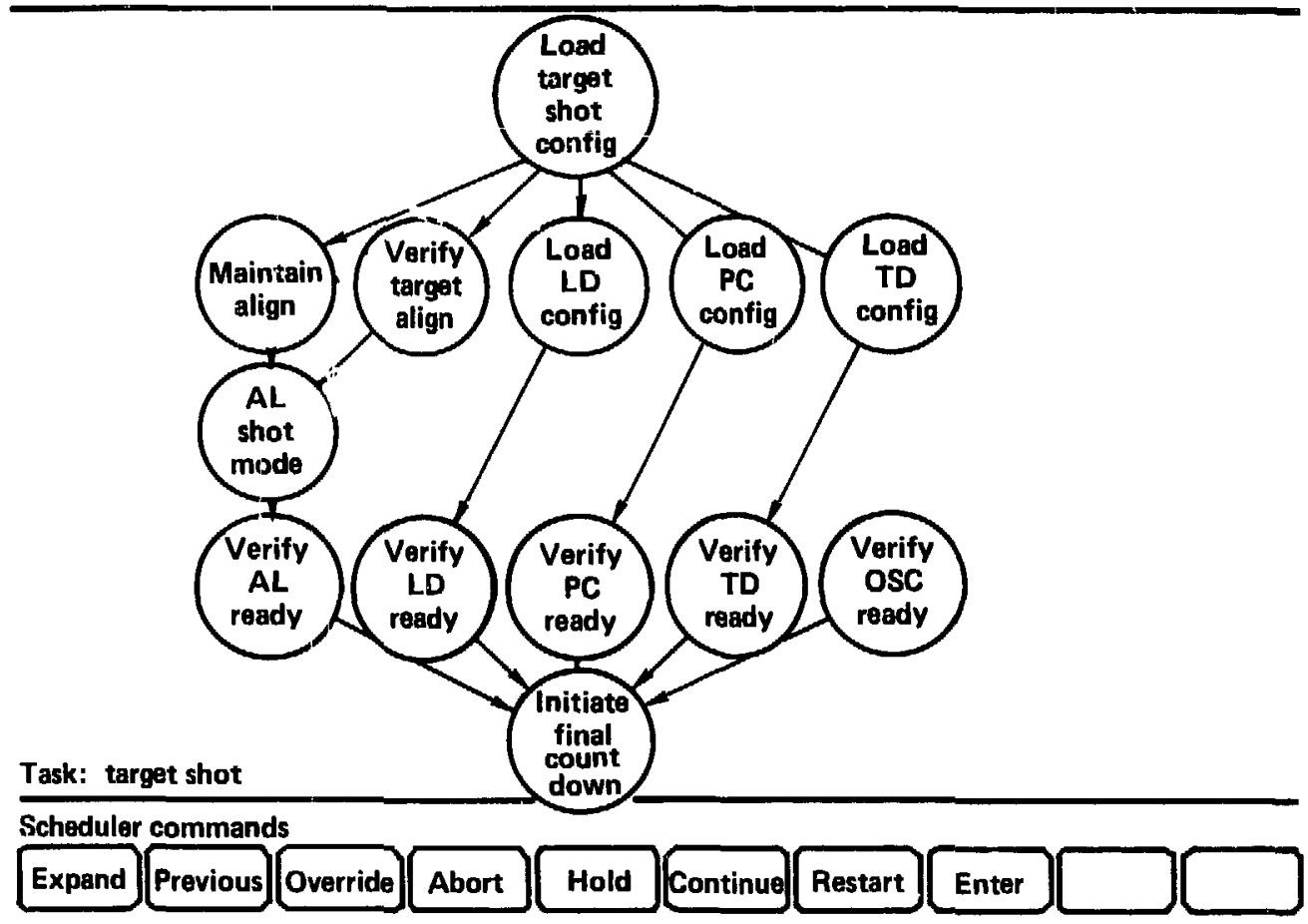

Fig. 2. Control frame display for a data-flow diagram. The data-flow diagram is represented here by circles and arrows. The circles represent tasks to be performed, and the arrows represent task dependencies. $\mathbf{A}$ task can execute when all its input dependencies have been completed. When a task is completed, it satisfies all its output dependencies, possibly causing other tasks to be executed. In this figuro, "AL shot mode" can execute after both "Maintain align" and "Verify align" are completed. When "AL shot mode" completes, "Verify AL" can execute. The "buttons" at the bottom of the figure perform commands related to the data-flow diagram.

Table 1. Task status.

\begin{tabular}{ll}
\hline \multicolumn{1}{c}{ Status (color) } & \multicolumn{1}{c}{ Definition } \\
\hline In progress (yellow) & Task started but not completed. \\
Not started (blue) & Task nut started. Input dependencies have not been satisfied. \\
Complete (successful) (green) & Task successfully completed. Outpul dependencies are satisfied. \\
$\begin{array}{l}\text { Requires operator input } \\
\text { (blinking white) }\end{array}$ & Task requires opirator input. Operator notifies scheduler when he completes the task. \\
Complete (overridden) (magenta) & Task overridden (stopped), and all output dependencies are satisfled. \\
Holding (blinking yellow) & Task holding due to operator command or problem detected by a subsystem. \\
Aborted (red) & Task aborted due to problem detected by a subsystem, or the operalor manually aborted it. Task \\
& may not continue from where it was aborted, but must be reset. \\
\hline
\end{tabular}


The operator controls the operation of the scheduler with the control buttons at the bottom of the control frame. The "expand" button enables the operator to expand any task bubble that represents a data-flow diagram of subtasks. The "previous" button contracts a data-flow graph into one bubble in its direct ancestor's data-flow graph in the tree and causes the direct ancestor's data-flow graph to be displayed. The "hold" button, which prevents the execution of a task until the "continue" button is pressed, can be used in case of problems or to prevent a task from being initiated until another task is performed. The "override" button-which tells the scheduler to ignore the task, mark it as complete, and execute subsequent tasks-is used only when the operator knuws more than the control system about the tasi's in question. If the operatur uses the "abort" button, the affected task can be restarted only by using the "restart" button.

When a problem is found, the operator may have enough information to deduce the problem directly, or he can request the appropriate subsystem status display to report the problem. Manual controls available through the subsystems are used to make repairs and adjustments, after which the operator can restart or override associated tasks to continue the sequence.

\section{Final countdown}

After verifying that the system is ready for a shot, the operator initiates the presequence phase of the countdown. During this phase, the operator executes tasks that must be done within a few minutes of the shot, but do not need to be done at a precise time. Such tasks include disabling instrumentation calibrations for detectors that drift over time.

Immediately after completing the presequence phase, the operator initiates the sequencer to schedule the remaining tasks that require accurate sequencing and timing. The sequencer starts the countdown time at minus 2 min, counting down in 1-s increments to zero, when the laser is fired, and continuing to plus $2 \mathrm{~min}$. For more information about sequencer timing, refer to the section on the power-conditioning subsystem.

Since the tasks in the presequence phase require an indefinite amount of time to complete, doing them without time allocations allows the total countdown time to be compressed. This minimizes loss of productive work time, because personnel must leave the laser area for safety reasons while the countdown is active.

\section{Operational interlocks}

Many tasks cannot be prerformed in parallel because of incompatible demands on system hardware. One obvious example: the laser cannot be aligned and fired at the same time or in reverse order. Therefore, operational interlocks are used in the scheduler to ensure that certain tasks are performed neither simultaneously nor out of sequence.

\section{Scheduler software components}

Figure 3 shows the scheduler programs and their relationship to other software components. Scheduler programs communicate among themselves and with other software components through memory shared by the three VAX control computers. Functions of these programs are as follows.

SCHED maintains the data-flow diagrams, interprets them to determine when tasks are executable, and then commands the subsystems to start their tasks. After the subsystems return taskstatus information to SCHED, it updates its stored task information and displays the latest status on the data-flow diagrams. When a new data-flow diagram is desired, SCHED reads the file containing it into shared memory and then begins its execution.

CNTDWN, the final countdown software, coordinates the control systems at shot time. It initiates the system-readiness checks and holds the countdown if any problems are detected in the system. CNTDWN sends signals to each of the subsystems at specific times. The countdown does not continue until every subsystem responds favorably to those signals. This active message-passing ensures that subsystem software is functioning properly, which in turn ensures that subsystem hardware is being checked and is responding properly.

If a subsystem detects a problem that warrants holding the countdown, it sends a hold request to CNTDWN. A hold request consists of the request itself and the reason for the request. CNTDWN holds the countdown and displays the reason on the operator's console. The operator then issues commands to fix the problem and continue the countdown, or override the problem and continue the countdown, or abort the countdown.

To $\log$ these events for future reference, SCHED and CNIDWN send commands to LOGGER, which maintains the scheduler's log of 
major events and creates log files for archiving. Investigators or reviewers can examine the log at any time to determine what events took place at any particular time.

INCONT provides the interface to the operator. It services menu selections sent by the console-graphics-system software and sends corresponding commands to SCHED, CNIDNN, and LOGGER. It also sends status stored in shared memory to the console screens. As each console has its own copy of INCONT, all consoles can be used with the scheduler simultaneously.

Global shared memory is available to all Nova control VAX programs. The shot number, the type of shot, and the chains selected for the next shot are examples. of data used by many control programs. These data reside in the MA780 multiport memory shared by all control VAXs.

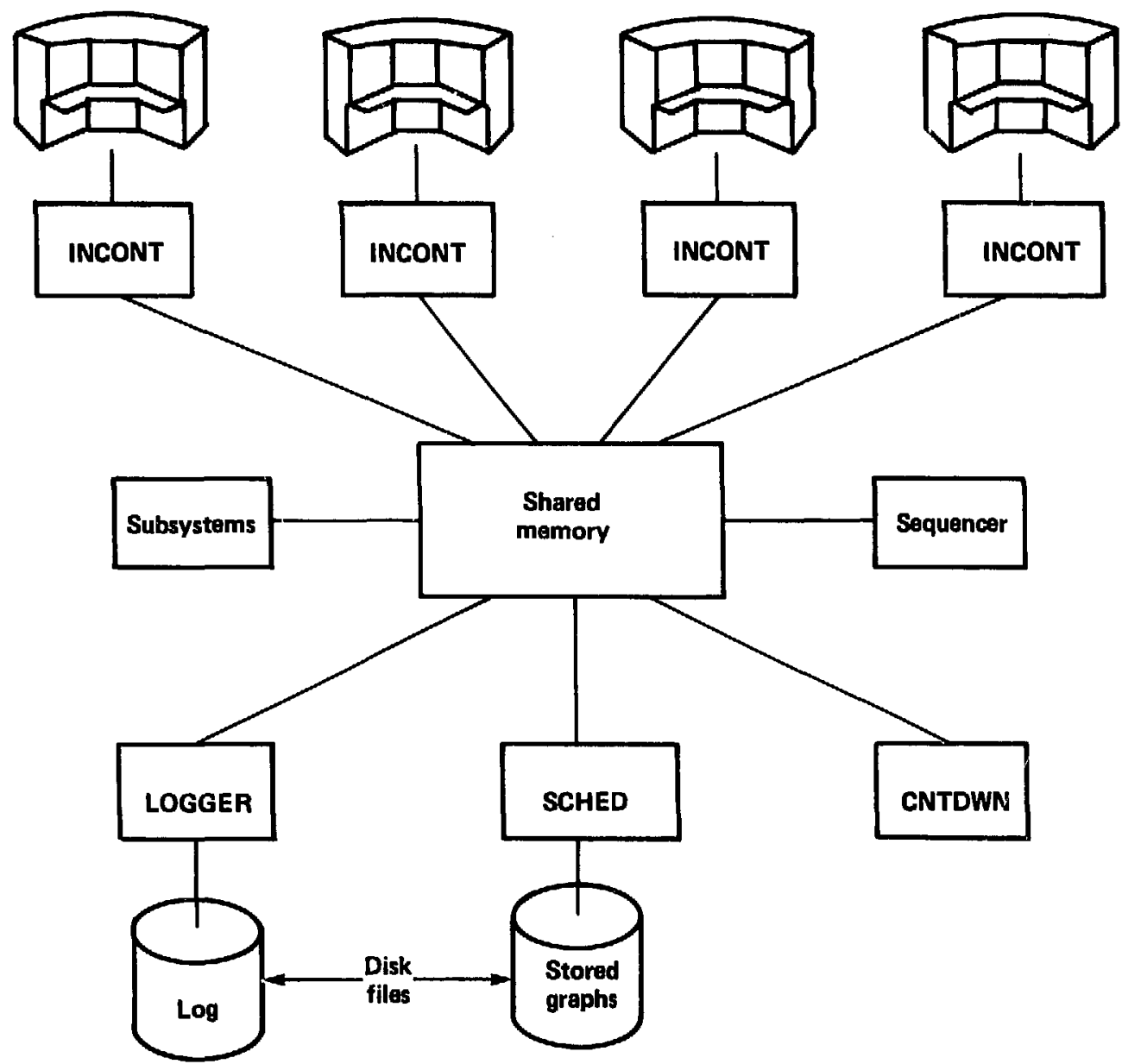

Fig. 3. Scheduler programs. Sch. -uler programs communicate among themselves and with the subsystems through shared memory. SCHED implements the scheduler algorithm. CNIDWN coondinates activities at final countdown time and starts the sequencer. The sequencer implements the one-second-resolution clock started two minutes before the laser is fired. INCONT provides the interface between the scheduler and the operator via the central controls console. LOGGER logs significant events to disk. 
Communication among the scheduler, finalcountdown software, and the subsystems is controlled by Nova Shared Memory (NSM). NSM uses global selections and event flags; both of which can be placed in the MA7BO memory. Processes waiting for data update can reside on any control VAX. Each process acectiated with global data always receives its own copy of the update information, regardless of which process receives it first. When a process awaiting data changes is added to the system, it is not necessary to change the communications code in any other program. The addition of the process is handled automatically by the NSM code residing in the other programs.

\section{Conclusion}

The Nova shot scheduler coordinates, at the highest level, the four large and diverse subsystems that operate the Nova laser facility. Modeling the tasks to be scheduled as a data-flow diagram allows the scheduler to schedule tasks in any appropriate order, as well as schedule any number of simultaneous tasks. (The effort involved in specifying the scheduler model has, in itself, helped to clarify the diverse conflicts involved in operating such a large facility.) Data-flow graphs are viewed and manipulated directly at the console and are easily changed, allowing rapid schedule changes for new laser experiments. Hierarchical data-flow graphs simplify large graphs into smaller ones. The scheduler unifies Nova's complex control systems to increase throughput and reduce the chances for error.

\section{Reference}

1. P. C. Treleaven, D. R. Brownbridge, and R. P. Hopkins, "Data-Driven and DemandDriven Computer Architecture," Computing Surveys 14(1) 93 (1982). 


\section{Index}

This index lists the subjects and authors to be found in published EE Quarterlies and EE Technical Reviews (including this issue). The number after an item indicates the year and issue in which the item was published. For example: Cross-borehole, 79-1, 81-3 means the subject is in the first issue of 1979 (report No, UCRL-50025-79-1) and in the third issue of 1981 (report No. UCRL-50025-81-3).

A

Ables, E., 80-1

Alexander, E., 75-1

Alpha emitters, long-lived, 80-1

Alrick, K., 75-1

Aluminum-air battery, 82-1

Ames, H. S., 76-2, 79-3

Analyses, automated chemical, 75-3

geological, 78-3

Analysis and man3gement, 79-3

Analyzer,

chlorine, 79-1

Dunegan 920, 77-1

radioisotope, 76-3

Anesthesia, 80-2

Antenna, unique directional, 81-3

Argus laser, 77-2

Arming, 77-3

Armstrong, G. H., 79-3

Artificial people, 80-3

Axelrod, M. C., 78-3

\section{B}

Baker, J., 76-1, 78-3

Balch, J. W., 75-2, 81-1

Barlow, O. M., 75-3

Barton, V., 75-3

Baseball-1l, 76-1

Battery, aluminum-air, 82-1

Behrin, E., 82-1

Bell, H. H., 79-2

Benge, W. L., 75-3

Bishop, S. R., 78-2

Bogdanoff, A., 76-1

Bourret, 5., 75-3

Bozorgamesh, H., 81-1

Bradley, R. T., 75-4, 76-2

Braille, 82-1

Brase, J., 84-3

Brazing furnace, induction, 75-1

Breitenbucher, P., 84-1

Brown, B. J., 79-1

Brown, D. R., 81-1

Bryant, R., 84-2

Buettner, H. M., 81-3

Burgess, T. J., 77-2

Bus, general purpose interface, $80-1$

Bus structure, 76-2

Butner, D. N., 77-3
C

CAIN, 76-2

CAMAC, 80-3, 83-3

CATV, 78-1

CMOS, 77-2

CTR, see Controlled thermonuclear

Calibration, automatic, 74-4

Candy, J., 82-2

Carbon dioxide sensor, 79-2

Casterson, R., 77-1, 83-1

Castleton, R. N., 84-1

Chau, H. H., 79-2

Chiorine analyzer, 79-1

Christie, D., 84-2

Ciarlo, D., 75-1, 81-1

Circuit diagnostics, 84-2

Clark, J. C., 77-2

Cleland, L. L., 77-1

Clendenen, L. D., 77-2

Coal, high speed spectroscopy for process control, 81-1

Coffield, F. E., 84-3

Communications cards, LSl-11, 84- $\bar{z}$

Communications link, fiber optics, 80-2

Computer model, 77-2

Control system, isolation, 78-1

Control systems, MFTF, 83-1

Controlled thermonuclear research, 76-1

Copper resistivity, 77-2

Coradetti, T., 84-1

Coutts, G. W., 75-1

Crates, CAMAC, 80-3

Criminal diversion of nuclear materials, 77-1

Criticality false alarm, 76-1

Cross-borehole, 79-1, 81-3

Cruise missile, computer-based testing of, 82-3

D

DASLL, 80-3

Danielli, F., 80-1

Data

acquisition, 77-1, 80-1

collection, 76-1

gathering, 76-1

handling, 79-3

low-level signal, 82-3

Davis, G. E., 81-2 
De Groot, T., 85-2

Deadrick, F. J., 75-2

Delay generator, 78-3

Detectors, time-resolved radiation, 75-2

Diagnostics, 77-1, 79- $-\mathbf{z}$

2XIIB 76-1

Digital techniques, high speed, 78-2

Distributed instrumentation control, 83-1

Drill press, 79-1

Druce, R., 84-2

Dye lasers, 78-2

\section{E}

Electron Cyclotron Resonant Heating, 84-3

EPA lab, 76-2

Editing, text, 77-3

Effluent characteristics, 75-3

Ekstrom, M. P., 75-1

Electro-optics, 76-3, 79-2

Electromagnetic cross-borehole, 79-1 railgun, $80-1$

Energy storage, 77-3

Equations-of-state, 79-2

Ewins, J. H., 75-2

Explosive, 79-2, 79-1

\section{F}

Fiber optics, 77-2, 80-2, 83-3

Fiber-optic data links, 81-2

Fiber-test facility, 77-1

Field-compression devices, 77-2

Fisher, E. R., 75-3

Flow cell analyzer, 75-1

Fluorometric, 78-2

Follow-up systems design, 82-3

Framing camera, 75-4 tube, 78-3

Francis, F. D., 80-3

Friesen, R. D., 76-1

Fuels, alternative transportation. 75-4

Fusion, laser, 77-2

Fuzing, 77-3

G

Gadd, T. J., 76-3

Gages, talking, 83-1

Gas-pressure, diagnostic system, 81-2

General Purpose Interface Bus, see GPIB

Geologic, 78-3

Geology, 79-1

Geophysical probing, 75-2

Goerz, D, , 79-1

Gordon, L., 84-2

Gozani, T., 81-1

GPIB, 80-1

Graphics, computer, 79-2

Gunn, R. C., 81-1, 82-3
H

Hasbrouck, R. T, 85-1

Hawke, R. S., 80-1

Herget, $C_{\text {, , 80-2, 82-2 }}$

Hersey, R. J., 76-1

High voltage test stand, 78-2

High-speed photography, 75-4

High-speed radiation camera, 75-1

Hill, C., 79-3

Holloway, F. W., 75-2

Hollstein, R, B., 77-2

Huebel, J. R., 75-4

Huen, T., 75-2

Hunt, D. N., 83-1

Hunt, G. F., 80-2

Hydrodiagnostic, 77-3

Hydrodynamic test data, 79-3

Hydrogen, compressed, 80-1

I

IC. 81-1

ICRF, 84-3

Image intensifiers, pulsed, 75-4

Image processing, digital, 75-1

Imaging, 77-1

Imaging gamma-ray cameras, tumor, 75-2

Imperial Valley, California, 78-3

Induction brazing furnace, 75-1

Interferometer phase detection, 78-2

Interferometer, microwave, 75-1

Interlocks, CAMAC-based, 83-3

Ion-implantation, 81-1

Isocon camera, 80-3

J

Jaroska, M. F., 75-4, 77-3, 82-1

Jensen, C. W., 75-3

Johnson, B. C., 76-3

Johnson, G. W., 84-1

$\mathbf{K}$

Kalibjian, R., 78-3, 75-4

Kaser, J. B., 78-3

Kaufman, A. M., 81-2

Koo, J. C., 84-3

Krammen, J., 85-2

Krausse, G. J., 85-1

Kuramoto, A., 79-3

L

LGF data acquisition system, 84-1

LSI development, 75-2

LSI-11, 84-2

Lager, D. L., 75-2, 80-2

Laine, E. F, 81-3

Language aids, 77-2 
Large optics diamond turning machine, 84-1

Laser, 75-2, 75-4, 76-3, 77-2, 78-1, 7)-2, 78-2, 80-2

optics, 83-1

Lathe, LeBlond, 79-1

Latorre, V. R., 80-1

Lawson, J. C., 77-3

LeBlond lathe, 79-1

Lee, F. D., 75-3, 79-2

Leighton, J., 77-1

Lightning vulnerability of nuclear explosive test systems, 85-1

Liston, D., 77-1

Long, J. R., 76-2

Low-level signal data acquisition, 82-3

Lytle, R. J., 75-2, 79-1

\section{M}

MACRO2, 81-1

MFECC, see NMFECC

MFTF, 81-2, 81-3, 82-1, 82-3, 83-3

MFTF-B power supply, computer model, 85-1

MGX, 83-3

MOS fast-switching techniques, 85-1

MOS time-resolved radiation detectors, 75-2

MOS/LSI facility, 75-1

Machine-control system, programmable, 75-3

Macro, structured, 77-2

Magnet, superconducting, 82-3

Magnetic fusion, 76-1 energy, 78-3,

Magnetic mirror fusion, 78-1

Magnets, 79-1

Magnuson, W. G., 80-3

Maiten, M. S., 80-3

Maples, M. D., 75-3

Mass storage, 78-3

McConaghy, C., 75-1, 75-2, 81-1

McCue, H. K., 84-1

McGibbon, A. L., 76-3

McQuaid, I. H., 75-2, 79-2, 81-1

Meeker, D. J., 75-1

Mendell, D. S., 78-2

Message processing system. automated, 75-3

Microchannel plate $x$-ray spectrometer, ultrafast gating, 85-1

Microcomputer, 77-2, 78-2, 79-1, 82-3

Microcomputer, special issue, 75-3

Microcomputer system, modular, 75-3

Micropellet guidance, 76-1

Microprocessor, 79-2

LSI-11, 77-1

Microstructure fabrication, 81-1

Mihalka, A. M., 81-3

Miller, E. K., 75-2

Minicomputer, 77-3

bus structure, 76-2

D112, 77-1
Minor, E. G., 82-3

Monitor, fast scan, 78-1

Montoya, C., 82-3

Mooney, L. J., 78-1

Moore, T. L., 84-3

Morrison, J., 75-1

Mullenhoff, D., 81-3

Multiprocessor, debugging, 81-2

Myers, D., 85-2

\section{$\mathbf{N}$}

NAD system, 75-1

NC machine tool, see Numerically controlled

NC, see Numerically controlled

NMFECC, 77-1

NTS diagnostics, 75-1

NURE program, 78-3

National MFE computer network, see NMIFECC

Networking microcomputers, 80-1

Neutral beam, 82-1

injectors, 76-1

ion-density profile, 78-1

power supply, 78-1, 81-3

protection circuits, 83-3

Neutron monitoring, 76-1

Neutron, portable spectrometer, 80-2

Neutron-gamma field, 80-2

Newton, L. E., 76-2, 79-1

Niven, W. A., 82-1

Nova laser, 80-2, 84-2

Nova

Alignment control, 85-2

Beam diagnostics, 85-2

Central controls, 85-2

Pulse pnwer control, 85-2

Target diagnostics, 85-2

Shot scheduler, 85-2

Nuclear science data production facility, 83-1

Nuclear test, underground, 77-1

Nuclear waste repository, $81-2$

Numerically controlled machine tool, 76-2, 76-3

$\mathbf{0}$

O'Brien, D. W., 83-1

Octopus, line switching, 83-3

Oicles, J. A., 85-1

Oil shale, 82-1

Olk, L. B., 75-1

Olken, H., 75-3

Optics, high power laser, 83-1

Oscilloscope,

dual raster, 77-3

fast-transient, 75-3

Oxidants, photochemical, 78-3

Ozarski, R., 85-2 
Pavel, G., 84-2

People, artificial, 80-3

Peratt, A. L., 75-2

Phase detection interferometer, 78-2

Photochemical oxidants, 78-3

Photodetector, 79-2

Photometer, a scanning reflection transmission, 83-1

Plasma diagnostics, 82-1

Pocha, M., 81-1, 84-3

Pockels cell, 76-3

Pollock, G. G., 76-1

Pomernacki, C. L., 79-1, 80-1

Portnoff, M., 82-2

Power supplies, 82-1

Pressure switch, sulnanosecond MOS, 75-4

Printed circuit board, 77-1

layout, 80-3

Pulse generator, 84-2

Pulse height analyzer, high speed, 82-1

Pulse power, 78-1

Pulsed image intensifiers, 75-4

\section{$\mathbf{R}$}

Rabbit, 75-3

Radiation camera, high-speed, 75-1

Radiation detectors, time-resolved, 75-2

Radioisotope analyzer, 76-3

Rail-gap switch, 84-2

Railgun, 80-1

Reacturs, 80-1

Recorder, on-board, 82-1

Recording, high-velocity, impact, 82-1

Rectifier transformer, 81-3

Relativistic beam, 77-2

Retorts, 82-1

Ring seating, 84-1

Robinson, W. F., 83-1

Ronchetto, J. J., 82-1

Rotating turbine camera controller, 84-1

Rotter, M. D., 81-2, 83-3

Route profile analysis, 81-3

Rufer, R. P., 78-1

Rupert, P. R., 78-1

\section{$\mathbf{S}$}

Safety, machining, 79-1

Safing, 77-3

Scarafiotti, J. J., 83-3

Scarpetti, R. D., 83-3

Sensor, carbon dioxide, 79-2

Severyn, J. R., 77-2, 80-2, 85-2

Sewall, N. R., 75-4, 77-1

Shaker control, 75-2

Shiva, 78-1

Shock-wave-velocity measurements, 75-4

Signal and Control Engineering, 82-2

Smart, J., 85-2

Smith, B. H., 76-1

Smith, M. E., 78-2
Smith, R. J., 77-1

Software debugging tool, 81-2

Solder-joint reliability, 76-1

failures, 76-1

Spann, J. M., 75-3, 81-2

Sparks, D. F., 80-3

Spectrometer,

gamma ray, 76-3,

portable neutron, 80-2

$x$-ray, 76-3

Spectroscopy, 81-1

Sphere-winding machine, 75-4

Spracklen, H. P., 82-1

St. Leger-Barter, G., 75-1, 75-4

Steinmetz, L. L., 75-1, 79-2, 78-2

Strauch, M. S., 83-3

Streak camera,

isocon, 80-3

$x$-ray, 75-1

Substation, 230-kV, 81-3

Superconducting cable, 79-3

Surgery, 80-2

Switches, high speed, 84-3

Switching, high power, 76-2

Synthesizer, voice, 83-1

$\mathbf{T}$

TMX, see Tandem Mirror Experiment

Tandem Mirror Experiment, 79-1, 81-2, 84-3

Technology transfer, 75-3

Temperature control, 75-1

Test, nuclear, 79-2

Testing, short circuit, 81-3

Text editing, 77-3

Thermometry, 82-1

Thomson scattering, 75-2

Three-dimensional images, 75-4

Thrust area, 82-2

Time-resolved radiation detectors, 75-2

Transient electromagnetics measurement, 75-2

high-speed recording of, 81-1 output current, 82-3 recorder, 79-3

Transportation, alternative fuels, 75-4

Transuranics, 80-1

Tumor, 75-2

Twogood, R., 82-2

Tyler, G. C., 76-1

$\mathbf{U}$

U.S. Postal Service, 81-3

Ultrasonic, 82-1

inspection of superconducting cable, 79-3

test bed, 84-3

Underground caverns and tunnels, 81-3

V

Van Arsdall, P., 85-2

Van Ness, H., 82-3 
Victor, R. A., 76-2, 79-3

Video playback facility, 81-1

Vogtlin, G., 84-2

Voice synthesizer, 83-1

$\mathbf{W}$

Warhus, J., 82-3

Water samples, 79-3

Waugh, A. F., 78-1

Weapon control, 75-2

Weiskamp. T., 75-3

Wentz, D. L., 78-3

Werner, R. E., 82-1

Whelan, H. A., 76-3

Whitham, K., 77-3

Wiedwald, J. D., 85-1

Willows, J. L., Jr., 82-1

Wilson, J. H., 81-3

Wind calculations, NIS, 75-3

Woodruff, J., 82-1 $\mathbf{x}$

X-ray

medical, 75-4

monitor and control, 79-3

pulse calorimeter, 75-2

spectrometer, 76-3, 85-1

streak camera, 75-1

$\mathbf{Y}$

Young, R. H., 75-3

$\mathbf{z}$

Zoret, R. E., 75-4

Numeric

2XIIB diagnostics, 76-1 